\title{
IFU spectroscopy of Southern Planetary Nebulae V: Low-ionization structures
}

\author{
A. Ali, ${ }^{1,2}$ and M.A. Dopita, ${ }^{3 *}$ \\ ${ }^{1}$ Astronomy Dept, Faculty of Science, King Abdulaziz University, Jeddah, Saudi Arabia. \\ ${ }^{2}$ Department of Astronomy, Faculty of Science, Cairo University, 12613, Giza, Egypt. \\ ${ }^{3}$ Research School of Astronomy and Astrophysics, Australian National University, Cotter Rd., Weston ACT 2611, Australia.
}

\begin{abstract}
In this 5th paper of the series, we examine the spectroscopy and morphology of four southern Galactic planetary nebulae Hen 2-141, NGC 5307, IC 2553, and PB 6 using new integral field spectroscopy data. The morphologies and ionization structures of the sample are given as a set of emission-line maps. In addition, the physical conditions, chemical compositions, and kinematical characteristics of these objects are derived. The results show that PB 6 and Hen 2-141 are of very high excitation classes and IC 2553 and NGC 5307 are mid to high excitation objects. The elemental abundances reveal that PB 6 is of Type I, Hen 2-141 and IC 2553 are of Type IIa, and NGC 5307 of Type IIb/III. The observations unveil the presence of welldefined low-ionization structures or "knots" in all objects. The diagnostic diagrams reveal that the excitation mechanism of these knots is probably by photo-ionization of dense material by the nebular central stars. The physical analysis of six of these knots show no significant differences with their surrounding nebular gas, except their lower electron densities. In spite of the enhancement of the low-ionization emission lines of these knots, their chemical abundances are nearly comparable to their surrounding nebulae, with the exception of perhaps slightly higher nitrogen abundances in the NGC 5307 knots. The integrated spectrum of IC 2553 reveals that nearly all key lines that have led researchers to characterize its central star as a weak-emission line star type are in fact of nebular origin.
\end{abstract}

Keywords: ISM: abundances-planetary nebulae: individual: Hen 2-141-planetary nebula: individual: NGC 5307-planetary nebula: individual:IC 2553-planetary nebula: individual: NGC 5307-planetary Nebula: individual: PB 6-planetary nebulae.

\section{Introduction}

The study of planetary nebulae (PNe) and their central stars (CSs) provides valuable constraints on the evolution of both low and intermediate mass stars from the asymptotic giant branch (AGB) to the white dwarf phase of evolution. Almost all spectroscopic studies of $\mathrm{PNe}$, up to now, have applied long-slit spectroscopic technique in the analysis. Such measurements are confined to a small portion of the entire nebula depending on the slit size and position. By contrast, measurements using the integrated field unit (IFU) technique cover the entire nebula (assuming that its angular size is smaller than the instrument field of view).

In this article, we continue our journey to analyze the IFU spectra for southern Galactic PNe using the Wide Field Spectrograph (WiFeS) instrument mounted on the $2.3 \mathrm{~m}$ ANU telescope at Siding Spring Observatory. Precise nebular analysis and modelling depend on

*E-mail: afmali@kau.edu.sa; Michael.Dopita@anu.edu.au an understanding of their integrated spectra and spatial structures. The data cubes generated by the WiFeS device help us not only to study the PNe physical and kinematical properties, but also to explain the internal flux distributions, ionization structures, and overall morphologies. More details regarding the advantages of using the IFU spectroscopy compared to the long-slit spectroscopy have been presented in earlier papers, see Ali et al. (2016) and Basurah et al. (2016).

The first uses of the IFU spectroscopy in this field was by Monreal-Ibero et al. (2005) and, a little later by Tsamis et al. (2007). More recently, Ali et al. (2016) have used the WiFeS instrument to extract integrated spectra of the Galactic planetary nebulae: M3-4, M 3-6, Hen 2-29, and Hen 2-37. These observations allowed extraction of the spectrum of the central star (CS) in the M 3-6 nebula. This was revealed to be H-rich and of the spectral type O3 I(f*). Further, they found most of the recombination lines which used previously to classify the CS as a weak-emission line star (WELS) type arise 
from the nebula rather than from its CS. Basurah et al. (2016) found another four examples of such misclassification of PNe CSs as WELS type, and provided detailed nebular analysis and modeling for the highly excited PNe: NGC 3211, NGC 5979, My 60, and M 42. Ali et al. (2015) extracted the integrated spectrum of the large, evolved, and interacting planetary nebula (PN) PNG342.0 - 01.7. The full spatial extent of the object required a mosaic of nine observing WiFeS frames.

The present article sheds light on the morphology and spectroscopy of another four southern PNe that are associated with the presence of low-ionization structures (LISs) "knots". Small-scale, low-ionization features in planetary nebulae has been considered by Balick et al. (1998). Gonçalves (2004) estimated that $10 \%$ of the Galactic PNe have associated LISs. In an earlier paper, Gonçalves et al. (2001) listed 50 PNe occupy different kinds of small-scale LISs such as jets, tails, filaments, and knots. From the observation of these LISs, Gonçalves (2004) summarize their characteristics as follows:

1. There is no preferred distribution for LISs amongst the different $\mathrm{PNe}$ morphological classes.

2. These structures do not display a density contrast with respect to the main nebular shell, suggesting that both are at the same pressure.

3. Most of the LISs studied until 2004 are photoionized.

4. Sometimes, they show faster expansion than the main PNe components, but sometimes expand the same velocity.

5. LISs appear as pair of jets, jet-like features, knots, filaments or as isolated systems.

In the specific case of fast, bipolar or jet-like ejection of LISs, these were termed fast-moving low-ionisation emission regions or FLIERs by Balick and co-workers (Balick et al. 1987, 1993, 1994), and these have variously been interpreted as jet shocks in a photoionised medium (Dopita 1997), recombination regions in a mass-loaded jet (Dyson \& Redman 2000), or as "stagnation knots" (Steffen et al. 2002). However, in other cases, the origin of the knots is more likely to be found in the late AGB mass ejection phase. Miszalski (2009) found LISs appear to be quite common amongst post common-envelope $\mathrm{PNe}$ and hence they strongly suggest binary origin of LISs. Recently Akras \& Gonçalves (2016) showed that the electron temperatures and chemical abundances of LISs observed in five PNe are comparable to all nebular components, whereas the electron density is systematically lower in LISs. They argue that the main excitation mechanism of LISs is due to shocks, while that of the other nebular components is due to photoionization.
Table 1 A summary of the observing log.

\begin{tabular}{llccc}
\hline Object & PNG number & $\begin{array}{c}\text { No. of } \\
\text { frames }\end{array}$ & $\begin{array}{c}\text { Exposure } \\
\text { time }(\mathrm{s})\end{array}$ & Airmass \\
\hline Hen 2-141 & PN G325.4-04.0 & 6 & 300 & 1.13 \\
NGC 5307 & PN G312.3+10.5 & 6 & 300 & 1.07 \\
IC 2553 & PN G285.4-05.3 & 3 & 600 & 1.12 \\
& & 2 & 300 & 1.4 \\
PB 6 & PN G278.8+04.9 & 3 & 50 & 1.13 \\
& & 1 & 300 & 1.14 \\
\hline
\end{tabular}

The main goal of this paper is to study the physical conditions, chemical composition, kinematical characteristics, ionization structures, and morphologies of four Galactic PNe: Hen 2-141 (PN G325.4-04.0), NGC 5307 (PN G312.3+10.5), IC 2553 (PN G285.4-05.3) and PB 6 (PN G278.8+04.9), and their associated knots, with the aim of helping to elucidate the origin of the knots, and their relationship with the main nebular structures.

The morphologies and structures of these PNe have been previously described in a number of articles. Narrow-band images of Hen2-141, in $\mathrm{H} \alpha$, [N II] and [O III] emission lines, show a bipolar morphology with the presence of two symmetrical knots along its polar axis (Corradi et al. 1996). Livio (1997) assign a point symmetric morphological class for NGC 5307. The morphology of IC 2553 was described as elongated inner shell, surrounded by a group of knots, with almost a spherical outer shell (Corradi et al. 2000). Finally, PB 6 reveals a relatively circular double shells in which internal structures appear as a set of knots (Dufour et al. 2015)

There have been few previous spectroscopic studies of these PNe, and almost all rely on the long-silt and échelle techniques. Costa et al. (1996) have determined physical conditions and chemical composition for Hen 2-141. Milingo et al. (2002a,b) provide detailed spectroscopic study for Hen 2-141 using a long-slit spectrum covering the range from 3600 to $9600 \AA$. Ruiz et al. (2003) have analyzed the physical conditions and chemical abundances for NGC 5307 using échelle observations in the spectral range $3100-10360 \AA$. Line fluxes and some nebular parameters of IC 2553 were determined by Gutierrez-Moreno et al. (1985). Later, Perinotto (1991) analyzed the chemical composition of IC 2553 and determined its elemental abundances. Pena et al. (1998) have presented spatially resolved long-slit spectra for $\mathrm{PB} 6$ to construct a photo-ionization model for the object.

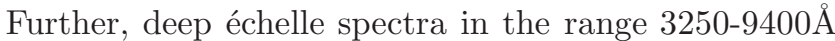
have been used to study the physical conditions, chemical composition, and abundance discrepancy problem in PB6 by García-Rojas et al. (2009). Finally, Henry et al. (2015) have used the HST/STIS spectra to study PB 6 and constructing photo-ionization model to predict the mass of its parent star.

This paper is structured as follows. The observations and data reduction are given in Section 2. Line fluxes, excitation properties, discussion of the excitation mech- 
anism of the knots, and their derived chemical abundances are discussed in Section 3. The kinematical characteristics are explored in Sections 4. Section 5 provides the morphologies as well as a general discussion for the results of the four nebulae and their knots. The misclassification of the CS of IC 2553 as a weak emission-line star (WELS) is demonstrated in Section 6, and the conclusions are given in the final Section 7 .

\section{Observations \& data reduction}

The IFU data cubes of the southern planetary nebulae Hen 2-141, NGC 5307, IC 2553, and PB 6 were obtained over two nights of 2013 March 28 and 30 using WiFeS instrument mounted on the $2.3 \mathrm{~m}$ ANU telescope at Siding Spring Observatory. This dual-beam image slicing integral field spectrograph and its on-telescope performance are described by (Dopita et al. 2007, 2010). It provides a $25 \operatorname{arcsec} \times 38$ arcsec field of view at spatial resolution of $1.0 \operatorname{arcsec} \times 0.5 \operatorname{arcsec}$ or $1.0 \operatorname{arcsec} \times$ $1.0 \mathrm{arcsec}$. The observed data provide low spectral resolution $(\mathrm{R} \sim 3000)$ that corresponds to a full width at half-maximum $(\mathrm{FWHM})$ of $\sim 100 \mathrm{~km} \mathrm{~s}^{-1}(\sim 1.5 \AA)$ for the blue spectral region $3400-5700 \AA$, and high spectral resolution $(\mathrm{R} \sim 7000)$ that corresponds to a FWHM of $\sim 45 \mathrm{~km} \mathrm{~s}^{-1}(\sim 0.9 \AA)$ for the red spectral region 5500 $7000 \AA$. A summary of the spectroscopic observations is given in Table 1.

All the data cubes were reduced using the PYWIFES data reduction pipeline (Childress et al. 2014). The STIS spectrophotometric standard stars HD 111980 and HD 074000 were used to calibrate the flux intensities, while the $\mathrm{Cu}-\mathrm{Ar}$ arc lamp, with 40s exposures during the night, was used to calibrate the wavelength scale. The telluric absorption features arise from atmospheric oxygen and water vapour molecules were removed from the observations utilizing the B-type telluric standard HIP 54970 and HIP 66957 with the spectrophotometric stars as a secondary standard. The final data cubes were treated from the effects of cosmic rays, sky background, and instrumental sensitivity in both the spectral and spatial directions.

\section{Physical and chemical analysis}

\subsection{Line fluxes and excitation properties}

The global spectra of the PNe set were extracted from their specific data cubes using QfitsView software (which is a fits file viewer using the QT widget library developed at the Max Planck Institute for Extraterrestrial Physics by Thomas Ott). The red spectra were re-scaled, by a factor $\sim 1-2 \%$, to compensate the continuum level of blue spectra using the emission lines in the common spectral region $(5500-5700 \AA)$. Emissionline fluxes and their uncertainties were measured, from the final combined, flux-calibrated blue and red spectra, using the ALFA code (Wesson 2016). This code uses a group of emission lines which are expected to be present to construct a synthetic spectrum. The parameters used to build the synthetic spectrum are developed by a genetic algorithm. Uncertainties are estimated using the noise structure of the residuals.

The Nebular Empirical Abundance Tool (NEAT; Wesson et al. (2012)) was applied to derive the interstellar reddening coefficients and subsequent plasma diagnoses. The line intensities were corrected for reddening applying the extinction law of Howarth (1983). The reddening coefficient $c(H \beta)$ was determined from the ratios of the Hydrogen Balmer lines (assuming Case B at $T_{e}=10^{4} \mathrm{~K}$ ), in an iterative method. In Table 2, we compare the derived reddening coefficients and $\mathrm{H} \alpha$ and $\mathrm{H} \beta$ fluxes, on a log scale, with those given in the literature. In general, our results agree well with other studies, except slightly higher reddening coefficients derived for Hen 2-141 and PB 6.

In addition to the global spectra of the PNe sample we able to extract the integrated spectra of six knots, three belong to NGC $5307\left(\mathrm{~K}_{\mathrm{NW}}, \mathrm{K}_{\mathrm{SW}}, \mathrm{K}_{\mathrm{SE}}\right)$, two belong to IC $2553\left(\mathrm{~K}_{\mathrm{NE}}\right.$ and $\left.\mathrm{K}_{\mathrm{SW}}\right)$, and one associated with PB $6\left(\mathrm{~K}_{\mathrm{SE}}\right)$. In subsequent discussions, we will consider only these knots. Results relating to the knots should be taken with caution because their extracted spectra may be contaminated by the surrounding nebular gas. A rough estimate was made to the probable effect of the surrounding nebular emission to the knots emission that studied in the paper. The results show the contamination of the surrounding gas to NGC 5307 knots are in the range 10-17\%, in IC 2553 knots $14-19 \%$, and in PB6 knot 15-18\%. In all knots we see enhancement of the low ionization line fluxes of ([O I], [O II], $[\mathrm{N} \mathrm{II}]$, and $[\mathrm{S} \mathrm{II}]$ ) and a diminishing of the relative $\mathrm{He}$ II $4685 \AA$ compared with their parent nebulae. The list of PNe line fluxes and their associated knots are given in the Tables A1 and A2 in the Appendix.

PNe excitation classes were determined following the Reid \& Parker (2010) scheme. He II $\lambda 4686 / \mathrm{H} \beta$ line ratio probably provides the best indication for the nebular excitation class (EC). The He II $\lambda 4686$ line disappears completely in the spectra of low excitation PN $(\mathrm{EC}<5)$. Reid \& Parker $(2010)$ remarked that at EC $\geq 5[\mathrm{O} \mathrm{III}] / \mathrm{H} \beta$ line ratio increasing to some degree with nebular excitation, therefore, they incorporate this ratio with the He II $\lambda 4686 / \mathrm{H} \beta$ line ratio in classifying $\mathrm{PNe}$ with $\mathrm{EC} \geq 5$. The results show very high excitation classes for PB 6 and Hen 2-141 and mid to high excitation classes for IC 2553 and NGC 5307 (Table 2).

\subsection{Excitation mechanism of LISs}

In order to examine the excitation mechanisms of the LISs studied here, we have used the diagnostics tech- 
Table 2 Reddening coefficients, observed $H \beta$ and $H \alpha$ fluxes, and excitation class.

\begin{tabular}{|c|c|c|c|c|c|c|c|}
\hline \multirow[t]{2}{*}{ Object } & \multicolumn{2}{|c|}{$c(H \beta)$} & \multicolumn{2}{|c|}{$\log \mathrm{F}(H \beta)$} & \multicolumn{2}{|c|}{$\log \mathrm{F}(H \alpha)$} & \multirow{2}{*}{$\begin{array}{c}\text { Excitation class } \\
\text { Reid \& Parker (2010) }\end{array}$} \\
\hline & This Paper & Literature & This paper & Literature & This paper & Literature & \\
\hline Hen 2-141 & $0.89 \pm 0.03$ & $0.76^{(1)}, 0.57^{(2)}$ & -11.78 & $-11.65^{(3)},-12.11^{(4)}$ & -11.05 & $-10.99^{(5)}$ & 11.0 \\
\hline NGC 5307 & $0.55 \pm 0.09$ & $0.59^{(1)}, 0.42^{(4)}$ & -11.29 & $-11.18^{(3)},-11.61^{(4)}$ & -10.66 & $-10.66^{(5)}$ & 8.3 \\
\hline IC 2553 & $0.42 \pm 0.08$ & $0.49^{(1)}, 0.35^{(6)}$ & -10.88 & $-10.82^{(3)}$ & -10.29 & $-10.25^{(5)}$ & 8.2 \\
\hline PB 6 & $0.61 \pm 0.04$ & $0.52^{(1)}, 0.54^{(7)}$ & -11.93 & $-11.87^{(3)},-12.22^{(8)}$ & -11.28 & $-11.26^{(5)}$ & 13.6 \\
\hline
\end{tabular}

(1) Tylenda et al. (1992);(2) Costa et al. (1996); (3) Cahn et al. (1992); (4) Milingo et al. (2002a); (5) Frew et al. (2013); (6) Martin (1981); (7) Kaler et al. (1991);(8) Milingo et al. (2010)

nique given by Raga et al. (2008). The purpose of these diagnostic diagrams is to discriminate photoionized nebulae from shock-excited regions. Figure 1 shows that the position of all knots are compatible with them lying in the regime of photoionized plasmas. Thus, all the knots studied here are likely to be excited by their central stars. Raga et al. (2008) proposed the central star of $\mathrm{PN}$ is capable of producing emission line ratios analogous to those of shocked-excited nebulae provided that the local ionisation parameter is sufficiently low. These results are also consistent with the conclusion given by Gonçalves (2004) which also reported that most LISs systems are mainly photoionized. The other common diagnostic diagram produced by Sabbadin et al. (1977) also gives similar results to those given above. In addition, we found that almost all knots occupy the empirical zone of fast FLIERS in the Raga et al. (2008) diagnostic diagrams.

\subsection{Temperatures and densities}

The collisional excitation lines (CELs) and optical recombination lines (ORLs) identified in the PNe spectra are convenient for their use in both plasma diagnosis and for elemental abundances determination. The NEAT code uses the Monte Carlo technique to propagate the statistical uncertainties of the line fluxes to all other quantities, e.g. temperatures, densities, and ionic and elemental abundances. The emission lines detected in each PN spectrum cover a wide range of ionization states covering neutral species up to the fourth ionized species, e.g. [O I], [O II], [O III], [Ne IV] and [Ar V].

The electron temperatures and densities were determined from the NEAT code. The variety of spectral lines shown in the PNe spectra permitted us to determine the electron temperatures and densities from the low and intermediate-ionization zones. The nebular temperatures were determined from the line ratios [N II] $(\lambda 6548+\lambda 6584) / \lambda 5754$ and [O III] $(\lambda 4959$ $+\lambda 5007) / \lambda 4363$, while nebular densities were determined from the line ratios $[\mathrm{S} \mathrm{II}] \lambda 6716 / \lambda 6731$ and $[\mathrm{O}$ II] $\lambda 3727 / \lambda 3729,[\mathrm{Cl}$ III] $\lambda 5517 / \lambda 5537$, and [Ar IV] $\lambda 4711 / \lambda 4740$. In Table 3 , we list the densities, temper- atures, and their uncertainties for the $\mathrm{PNe}$ and their associated knots.

\subsection{Ionic and elemental abundances}

Applying the NEAT code, the ionic abundances of nitrogen, oxygen, neon, argon, sulfur, and chlorine can be derived from the CELs, while helium and carbon were calculated from the ORLs using the temperature and density relevant to their ionization zone. When several lines are observed for the same ion the average abundance was adopted. The total elemental abundances were determined from the ionic abundances using the ionization correction factors (ICFs) given by Delgado-Inglada et al. (2014). The ionic and total abundances for the PNe as well as knots studied in this paper are presented in Tables A3 and A4 in the Appendix.

The elemental abundances of the sample, in the form of $\log (\mathrm{X} / \mathrm{H})+12$ are presented in Table 4 and compared with those found in the literature. This comparison reveals good agreement with previous studies. The slight differences remaining can possibly explained due to the differences in observed line fluxes, as expected between long-slit and integral field datasets, as well as the different ICFs and different sources of fundamental atomic data.

The planetary nebula PB 6 is the only object in our sample is rich in helium $(\mathrm{He} / \mathrm{H} \geq 0.125)$ and nitrogen $(\log (\mathrm{N} / \mathrm{H})+12 \geq 8.0)$. It also has an $\mathrm{N} / \mathrm{O}$ ratio $\geq 0.5$. Therefore it is classified as Type I according to the original classification scheme proposed by Peimbert (1978), which probably indicates that the progenitor star of initial mass $\geq 4 M_{\odot}$. Hen 2-141 and IC 2553 both show an excess in nitrogen abundances $(\log (\mathrm{N} / \mathrm{H})+12 \geq 8.0)$ and have $\mathrm{N} / \mathrm{O} \geq 0.25$. Applying the Peimbert criteria as modified by Quireza et al. (2007) both objects can be classified as Type IIa. The elemental abundances of NGC 5307 show this object is both helium and nitrogen poor. Hence, the object is classified as being of Type IIb/III. 
Table 3 Temperatures and densities of the sample.

\begin{tabular}{|c|c|c|c|c|c|c|c|c|c|c|c|c|c|c|c|c|c|c|}
\hline \multirow[b]{2}{*}{ Hen 2-141 } & \multicolumn{3}{|c|}{$N_{e}[\mathrm{O}$ iI $]$} & \multicolumn{3}{|c|}{$N_{e}[\mathrm{~S}$ iI $]$} & \multicolumn{3}{|c|}{$N_{e}\left[\begin{array}{ll}\mathrm{Cl} & \mathrm{III}\end{array}\right]$} & \multicolumn{3}{|c|}{$N_{e}[\mathrm{Ar} \mathrm{IV}]$} & \multicolumn{3}{|c|}{$T_{e}\left[\mathrm{~N}_{\mathrm{NI}}\right]$} & \multicolumn{3}{|c|}{$T_{e}[\mathrm{O} \quad \mathrm{III}]$} \\
\hline & 1932 & $(1666)$ & $(-843)$ & 1355 & $(160)$ & $(-143)$ & 1184 & $(2291)$ & $(-1169)$ & 1347 & $(335)$ & $(-330)$ & 11107 & $(666$ & $(-622)$ & 12160 & $(92)$ & $(-92)$ \\
\hline NGC 5307 & 4422 & $(915)$ & $(-681)$ & 3639 & ( 2144$)$ & $(-1214)$ & 1388 & (1433) & $(-10)$ & 1449 & (285) & $(-285)$ & 13316 & ( 1189 & 90 ) & 12332 & ( 396 ) & $(-384)$ \\
\hline $\mathrm{K}_{\mathrm{NW}}$ & 3933 & ( 2510$)$ & $(-1326)$ & 1707 & ( 407 ) & $(-329)$ & 1327 & ( 3241$)$ & $(-1346)$ & 1510 & ( 813$)$ & $(-732)$ & 13093 & ( 681 & $(-614)$ & 12430 & (197) & $(-197)$ \\
\hline $\mathrm{K}_{\mathrm{SW}}$ & 3213 & $(632)$ & $(-528)$ & 3126 & ( 1133 ) & $(-767)$ & 2181 & ( 1214$)$ & $(-1006)$ & 1734 & $(432)$ & $(-396)$ & 13948 & ( 534 & $(-514)$ & 12302 & ( 380$)$ & $(-369)$ \\
\hline $\mathrm{K}_{\mathrm{SE}}$ & 3710 & ( 4633 ) & $(-1712)$ & 2954 & ( 813$)$ & $(-589)$ & 1708 & ( 3445$)$ & $(-1712)$ & 1662 & $(889)$ & $(-808)$ & 12827 & $(581$ & $(-637)$ & 12328 & ( 329$)$ & $(-329)$ \\
\hline IC 2553 & 5493 & ( 3113$)$ & $(-2617)$ & 3242 & ( 1382 ) & $(-874)$ & 4030 & ( 1397$)$ & $(-1189)$ & 4502 & (315) & $(-294)$ & 11033 & ( 739 & $(-998)$ & 10823 & ( 152$)$ & $(-152)$ \\
\hline $\mathrm{K}_{\mathrm{NE}}$ & 4233 & $(2876)$ & $(-1489)$ & 3054 & ( 1463$)$ & $(-889)$ & 3187 & ( 1296 ) & $(-1026)$ & 3456 & ( 1738$)$ & $(-1428)$ & 10719 & ( 598 & $(-591)$ & 10853 & ( 345$)$ & $(-345)$ \\
\hline $\mathrm{K}_{\mathrm{SW}}$ & 3314 & (1946) & $(-1092)$ & 1744 & ( 781$)$ & $(-539)$ & 2262 & $(960)$ & $(-782)$ & 3410 & ( 1854$)$ & $(-1590)$ & 10867 & ( 484 & $(-484)$ & 11012 & ( 344$)$ & $(-344)$ \\
\hline PB 6 & 2190 & (524) & $(-423)$ & 1765 & ( 244$)$ & $(-215)$ & 1932 & ( 1414 ) & $(-1992)$ & 1952 & ( 361 ) & $(-351)$ & 11909 & ( 397 & $(-397)$ & 14778 & (151) & $(-151)$ \\
\hline $\mathrm{K}_{\mathrm{SE}}$ & 1972 & $(1545)$ & $(-879)$ & 1513 & $(436)$ & $(-339)$ & 1881 & $(1387)$ & $(-1158)$ & 1642 & $(1636)$ & $(-1407)$ & 11154 & ( 423 & $(-423)$ & 13751 & (541) & $(-541)$ \\
\hline
\end{tabular}

Table 4 The total abundances, in the form of $\log (\mathrm{X} / \mathrm{H})+12$, of the sample and their knots compared with previous studies.

\begin{tabular}{|c|c|c|c|c|c|c|c|c|c|c|c|c|c|c|c|c|c|}
\hline \multirow[t]{2}{*}{ Element } & \multicolumn{3}{|c|}{ Hen 2-141 } & \multicolumn{6}{|c|}{ NGC 5307} & \multicolumn{4}{|c|}{ IC 2553} & \multicolumn{4}{|c|}{$\mathrm{PB} 6$} \\
\hline & PN & (1) & $(2)$ & $\mathrm{PN}$ & $\mathrm{K}_{\mathrm{NW}}$ & $\mathrm{K}_{\mathrm{SW}}$ & $\mathrm{K}_{\mathrm{SE}}$ & $(2)$ & (3) & $\mathrm{PN}$ & $\mathrm{K}_{\mathrm{NE}}$ & $\mathrm{K}_{\mathrm{SW}}$ & (4) & $\mathrm{PN}$ & $\mathrm{K}_{\mathrm{SE}}$ & (5) & $(6)$ \\
\hline $\mathrm{He} / \mathrm{H}$ & 11.04 & 11.11 & 11.08 & 11.00 & 11.04 & 11.08 & 10.95 & 11.00 & 11.00 & 11.04 & 11.15 & 11.04 & 11.04 & 11.23 & 11.18 & 11.26 & 11.23 \\
\hline $\mathrm{C} / \mathrm{H}$ & 8.83 & & & 8.55 & 8.28 & 8.68 & 7.81 & & 8.08 & 8.91 & 9.03 & 8.77 & 8.91 & 9.01 & & 8.92 & \\
\hline $\mathrm{N} / \mathrm{H}$ & 8.19 & 7.98 & 8.41 & 7.72 & 7.94 & 8.00 & 7.94 & 7.71 & 8.04 & 8.23 & 8.27 & 8.16 & 8.28 & 8.58 & 8.54 & 8.62 & 8.67 \\
\hline $\mathrm{O} / \mathrm{H}$ & 8.72 & 8.43 & 8.88 & 8.44 & 8.40 & 8.41 & 8.37 & 8.59 & 8.51 & 8.59 & 8.79 & 8.54 & 8.80 & 8.54 & 8.51 & 8.51 & 8.59 \\
\hline $\mathrm{Ne} / \mathrm{H}$ & 8.03 & 7.70 & 8.16 & 7.82 & 8.00 & 7.82 & 7.83 & 7.95 & 7.89 & 8.01 & 8.34 & 8.09 & 8.14 & 8.00 & 7.94 & 7.91 & 8.02 \\
\hline $\mathrm{Ar} / \mathrm{H}$ & 6.03 & 6.28 & 6.40 & 5.98 & 5.48 & 6.00 & 6.01 & 6.13 & 5.93 & 6.39 & 6.55 & 5.76 & & 5.95 & 5.96 & 7.59 & 5.97 \\
\hline $\mathrm{Cl} / \mathrm{H}$ & 5.25 & & 5.41 & 4.44 & 5.26 & 4.54 & 4.90 & 4.83 & 5.00 & 4.88 & 5.38 & 5.14 & & 5.22 & 4.96 & 5.26 & \\
\hline $\mathrm{N} / \mathrm{O}$ & 0.30 & 0.35 & 0.34 & 0.19 & 0.35 & 0.39 & 0.37 & 0.13 & 0.34 & 0.44 & 0.31 & 0.41 & 0.30 & 1.10 & 1.07 & 1.30 & 1.20 \\
\hline
\end{tabular}

References: (1) Holovatyy \& Havrilova (2005);(2) Milingo et al. (2002b); (3) Ruiz et al. (2003); (4) Perinotto

(1991); (5) Henry et al. (2015); (6) Perinotto et al. (2004); (7) Grevesse et al. (2010)

Table 5 Radial and expansion velocities of the sample.

\begin{tabular}{|c|c|c|c|c|}
\hline \multirow[t]{2}{*}{ Object } & \multicolumn{2}{|c|}{$R V_{\text {hel }}\left(\mathrm{km} \mathrm{s}^{-1}\right)$} & \multicolumn{2}{|c|}{$\mathrm{V}_{\exp }\left(\mathrm{km} \mathrm{s}^{-1}\right)$} \\
\hline & Ours & Literature & Ours & Literature \\
\hline Hen 2-141 & $-39 \pm 5$ & $-46 \pm 9.0^{1}$ & 28.0 & \\
\hline NGC 5307 & $38 \pm 3$ & $39 \pm 2^{1}$ & 22.1 & \\
\hline $\mathrm{K}_{\mathrm{NW}}$ & $33 \pm 6$ & & & \\
\hline $\mathrm{K}_{\mathrm{SW}}$ & $24 \pm 6$ & & & \\
\hline $\mathrm{K}_{\mathrm{SE}}$ & $12 \pm 6$ & & & \\
\hline IC 2553 & $27 \pm 3$ & $37 \pm 6^{1}, 31 \pm 4^{2}$ & 19.3 & \\
\hline $\mathrm{K}_{\mathrm{NE}}$ & $23 \pm 6$ & & & \\
\hline $\mathrm{K}_{\mathrm{SW}}$ & $17 \pm 5$ & & & \\
\hline $\mathrm{PB} 6$ & $51 \pm 4$ & $59 \pm 1^{1}$ & 28.7 & $34^{3}, 38 \pm 4^{4}$ \\
\hline $\mathrm{K}_{\mathrm{SE}}$ & $60 \pm 6$ & & & \\
\hline
\end{tabular}

\section{Kinematical characteristics}

The systemic velocities $\mathrm{RV}_{\text {sys }}$ of the sample were determined using the IRAF external package RVSAO (emsao task). Weighted mean systemic velocities were calculated from the seven emission lines: $\mathrm{H} \alpha$, [NII] $\lambda 6548$, [NII] $\lambda 6583$, [S II] $\lambda 6716$, [S II] $\lambda 6731$, He I $\lambda 6678$, and [O I] $\lambda 6300$. These lines lie in the red spectral region of high spectral resolution. The Heliocentric radial velocities $\mathrm{RV}_{\mathrm{Hel}}$, were calculated by correcting the $\mathrm{RV}_{\text {sys }}$ for the effect of Earth's motion, using the heliocentric correction factor given in the fits image header. In Table 5, we present our results compared with that of literature. The measurements of Hen 2-141 and NGC 5307 show good agreement with Durand et al. (1998) within the error range, while IC 2553 and PB 6 are slightly lower. The $\mathrm{RV}_{\mathrm{Hel}}$ of IC 2553 shows better agreement with the value derived by Corradi et al. (2000). The small differences appear in case of IC 2553 and PB 6 compared to the values of Durand et al. (1998) may be attributed to the different spectral resolutions as well as the fact that our measurements were extracted from integrated spectra. We also determined the $\mathrm{RV}_{\mathrm{Hel}}$ for all knots. In general, the knots show lower velocities compared to their associated nebulae, except the knot SE knot of $\mathrm{PB} 6$ which has a higher velocity. The $\mathrm{RV}_{\mathrm{Hel}}$ of the SW and SE knots of NGC 5307 have much lower velocity relative to the entire nebula. These results tend to suggest that the knots are best seen when they lie on the far side of the expanding nebula (and therefore seen with a redshift), which is consistent with them being dense partially-ionised structures photoionised on the side nearest the central star.

The expansion velocity is a key parameter in studying the PN evolution. The average expansion velocities of the sample were measured from the following emission lines: $\mathrm{H} \alpha,[\mathrm{NII}] \lambda 6548,[\mathrm{NII}] \lambda 6583$, [S II] $\lambda 6716$, [S II] $\lambda 6731$ and He I $\lambda 6678$ following Gieseking et al. (1986). Results are given in Table 5. Two measurements were also found in literature regarding the average expansion velocity of $\mathrm{PB} 6$. Both are slightly larger than our measurement. 


\section{Morphologies}

All objects in our sample reveal prominent lowionization regions in their nebular shells. To study these features and the PNe morphologies in general, we extract emission-line maps in species of differing ionisation potential from their WiFeS data cubes using the QfitsView software. In general, the ionization strength decreases outward from the CS due to the dilution and absorption of the stellar ionising radiation field. Thus, to reveal the ionization structures of these PNe, we build a composite color image for every PN by combining three lines of different ionization potential into one RGB image.

\subsection{Hen 2-141}

Corradi et al. (1996) have detected two radially symmetrical knots along the nebular polar axis and two symmetrical ansae along the major axis of Hen 2-141. This pair of ansae lies outside the WiFeS field of view. In Figure 2, we present two emission-line maps of Hen 2-141 in the lines of $\mathrm{H} \alpha$ (left panel) and [O II] $3726+3728 \AA$ (middle panel). The color bar on the right side of each map refers to the relative surface brightness of emission line depicted. The PN images in this figure and subsequent figures are oriented with north up and east to the left. The overall morphology of the object reveals a bipolar shape where the two lobes appear as two condensations of gas on the opposite sides of the nebular center, seen best in [O II] at the top and bottom of the image. There is also a pair of lower excitation knots at P.A. $\sim 134^{\circ}$ associated with the boundary of the inner elliptical shell.

In Figure 2 (right panel), we present a composite color image of the nebula in the RGB color system ([S II] (red channel), [S III] (green channel) and $\mathrm{H} \alpha$ (Blue channel). The purpose of this image is to probe the pair of knots and the overall ionization structures of the nebula. It is obvious the hydrogen gas (blue) is distributed throughout the PN and extends to the outer region. The two knots barely appear in magenta $(\mathrm{R}+\mathrm{B})$ color at the outer edges of the two lobes. The white $(\mathrm{R}+\mathrm{G}+\mathrm{B})$ color of the two lobes are due to the combination of neutral hydrogen with singly and doubly ionized sulfur gases. The lowest excitation regions appear red in this image.

The elemental abundances of the nebula agree well with the results of Holovatyy \& Havrilova (2005) and Milingo et al. (2002b), except the argon and sulfur elements which show slightly lower and higher abundances, respectively (Table 4). The determined N/O ratio are consistent with other works. The calculated radial velocity of the object agrees well with the value of Durand et al. (1998).

\subsection{NGC 5307}

NGC 5307 nebula has been classified as belonging to the point symmetric class: "the morphological components show point reflection symmetry about the center" (Livio 1997), based on the HST image taken by Bond et al. (1995). The same morphology was assigned by Gorny et al. (1997).

The [S III] and [S II] emission-line maps (Figure 3, left and right panels) of NGC 5307 reveal an overall rectangular shape with two symmetric pairs of knots on either side of the nebular center at P.A. $\sim 30^{\circ}$ and at P.A. $\sim 163^{\circ}$. The two pairs of knots which appear in Figure 3 are clearly visible in the HST image (Figure 6, Livio (1997)).

The four knots are very clear in magenta color of the composite RGB image (Figure 3, right panel). The NW and SE pair of knots are much brighter than the NE and SW pair of knots. The ionization stratification is evident in this image where the high-ionization helium gas (green color) is distributed in the center of the nebula, the neutral helium (blue color) lies throughout the nebula except the inner region, and the low-ionization oxygen (red color) is confined to the outer region of the nebula.

The plasma diagnostics given in Table 3 show consistency between the temperature of NW, SW, and SE knots and the nebula as a whole. However, the electron densities, $N_{e}[\mathrm{O} \mathrm{II}]$ and $N_{e}[\mathrm{~S}$ II $]$, of the knots are somewhat lower than the nebula. Furthermore, the spectroscopy of the knots suggest an enhancement in the nitrogen abundances compared to the nebula. The elemental abundances of NGC 5307 are compared with the results of Milingo et al. (2002b) and Ruiz et al. (2003) in Table 4. It is appropriate to mention here that the nebular spectra observed by those authors are much deeper than our IFU spectra, and cover the optical and near IR spectral range from 3100 to $10360 \AA$. Our derived abundances of almost all elements nearly match that derived by those authors, except for $\mathrm{Cl}$, which has lower abundances (Table 4). The derived $\mathrm{N} / \mathrm{O}$ ratio is higher than Milingo et al. (2002b) but lower than Ruiz et al. (2003). The radial velocity determined from IFU spectra is fully consistent with that of Durand et al. (1998).

\subsection{IC 2553}

A spatio-kinematic model was constructed for IC 2553 by Corradi et al. (2000). It reveals an elongated inner shell and a roughly spherical outer shell expanding with higher velocity relative to the inner one. They provide two narrow band images for IC 2553. The [O III] image delineates a roughly rectangular inner shell with two bulges along the short axis encircled by a faint outer shell, while the $[\mathrm{N} \mathrm{II}]$ image shows the inner shell is 
surrounded by few low ionization knots, a pair of which are located symmetrically to the CS.

Our emission-line maps of IC 2553 in $\mathrm{H} \alpha$ and [N II] $6583 \AA$ A lines were given in Figure 4. Both images reveal an overall elliptical morphology. The [N II] emission is more extended than $\mathrm{H} \alpha$ map with two protruberences at both sides of the minor axis. Further, this image revealed the presence of two knots which lie symmetrically along the major axis of the object at P.A. $\sim 32^{\circ}$. In the right panel of Figure 4, we present a composite RGB color image ([O II] (red channel), $\mathrm{H} \beta$ (green channel) and He II (blue channel). The two symmetrical knots are more evident here than in the $[\mathrm{N} \mathrm{II}]$ map. Both knots (in red colour) appear to be nearly isolated from the central regiont.We also note the presence of a faint low ionization structure located on the outer edge of the spherical region in the north-westerly direction.

The temperatures of NE and SW knots are both compatible with the entire nebula, while their densities are lower than the nebula. In general, the elemental abundances of IC 2553 agree well with the results of Perinotto (1991), except for the N/O ratio which appears much higher here. This is consistent with the fact that we include all of the [N II] - emitting zone in our observations. Furthermore, the abundances of the two knots are consistent with the nebula as a whole. As noted above, the radial velocities of both knots are smaller than the main shell.

\section{$5.4 \mathrm{~PB} 6$}

The ground-based narrow-band image of $\mathrm{PB} 6$ in [O III] filter (Dufour et al. 2015), shows two concentric nearly circular shells. These authors also present a higherresolution STIS/HST image which shows a complex system of knots inside the nebula. Figure 5 shows the morphology of $\mathrm{PB} 6$ as a roughly circular $\mathrm{PN}$ with faint extended halo appearing in the [N II] emission-line map (middle panel). This map reveals also three non symmetric knots inside the main ionized shell of the nebula. One of the knots has a tail-like extension. The [O I]He I- $[\mathrm{Ar} \mathrm{V}]$ composite RGB color image (right panel) shows the same features seen in the $[\mathrm{N} \mathrm{II}]$ image. The [Ar V] emission (blue color) fills the central region of the PN, while the He I emission (green color) is distributed at the outer region and partially extends into the inner region. Two bright knots are pronounced on the $\mathrm{E}$ side of the nebula in yellow $(\mathrm{R}+\mathrm{G})$ color. These seem to have fainter counterparts on the $\mathrm{W}$ side. In addition, another knot appears $\mathrm{N}$ of the nebular centre in magenta $(\mathrm{R}+\mathrm{B})$ color with a tail of magenta and green colors.

The expansion velocity of the entire nebula are slightly smaller than the values in literature (Table 5). The temperatures of the SE knot are slightly lower than the nebula as a whole. The densities of SE knot are marginally smaller than the nebula (Table 3 ). The total abundances of PB 6 are fully consistent with SE knot as well as the previous studies (Henry et al. (2015) and Perinotto et al. (2004)).

\section{Misclassification of IC 2553 central star}

Miszalski (2009) and Miszalski et al. (2011) claim that many of the weak-emission line CSs type are probably misclassified close binaries and their characteristic lines (C II at $4267 \AA$, NIII at $4634 \AA$ and $4641 \AA$, CIII at $4650 \AA$, CIV at $5801 \AA$ and $5811 \AA$ ) originate from the irradiated zone on the side of the companion facing the primary. The first evidence for the nebular origin of most key lines characterize the WELS type was given by Górny (2014) when he found these lines appear in a spatially extended region in the 2D spectra of NGC 5979. Basurah et al. (2016) declared that, for many objects, the WELS type may well be spurious. From WiFeS data of NGC 5979, M4-2 and My60, they showed that the characteristic CS recombination lines of WELS type are of nebular origin. They were re-classified the CSs of these nebulae as hydrogen rich $\mathrm{O}(\mathrm{H})$-Type . Ali et al. (2016) found a further example (M3-6) that further indicates the unreliability of the WELS classification. From a careful nebular subtracted spectrum of the CS in M3-6, they revised its classification as H-rich star of spectral type O3 $\mathrm{I}\left(\mathrm{f}^{*}\right)$. In addition, they show the emission of almost all proposed CS recombination lines as WELS type are spatially distributed over a large nebular area. Here, we present yet another example of misclassification of WELS type which raises the probability that most genuine cases of WELS originate from irradiation effects in close binary central stars.

The CS of IC 2553 has been classified as of the WELS type by Weidmann \& Gamen (2011). However, in Figure ??, we present emission-line maps of IC 2553 in

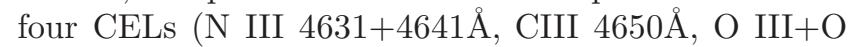
V $5592 \AA$, and C IV 5811 $\AA$ ) which are supposed to be of CS origin according to the definition of the WELS type. It is clear that the emission in all of these lines are spatially distributed over a large area of the PN, except for the C IV $5811 \AA$ line which may truly be of CS origin. Unfortunately we were unable to extract the CS spectrum from the available data cube of this PN, but it is clear that the WELS type classification is erroneous for this nebula.

\section{Conclusions}

We have studied the four southern PNe Hen 2-141, NGC 5307, IC 2553, and PB 6 using the integral field unit spectroscopy technique. We performed emissionline maps as well as composite color images to study the morphologies and ionization structures of these objects. The maps reveal the presence of low-ionization 
knots in all objects. Two knots appear in Hen 2-141, four in NGC 5307, two in IC 2553, and three in PB6. Furthermore, for first time, we shed the light to the spectroscopy of six knots associated with NGC5307, IC 2553, and PB 6. The derived physical and kinematical properties of these knots should taken with caution due to the probable contamination of these knots with their surrounding nebular gases. However, in general, the physical conditions and elemental abundances of the knots are agree with their accompanying nebulae, except the electron densities in the knots are systematically lower. Furthermore we noticed a slightly higher nitrogen abundances in the knots of NGC 5307 compared to the entire nebula. Those knots also have lower radial velocities than their parent nebulae, suggesting that they are dense part-ionised structures lying on the far side of the expanding nebula (and therefore seen with a redshift), and are have ionisation fronts photoionised on the side which lies nearest the central star.

The physical analysis of the entire nebulae indicate a medium to high excitation class for NGC 5307 and IC 2553 and very high excitation classes for Hen 2-141 and PB6. Their elemental abundances compare relatively well with those previously published in literature.

Another example for the misclassification of WELS type central stars was given here. The demonstrated emission-line maps of IC 2553 in the collisional characteristic lines of WELS type show that these spectral lines are spatially distributed over a wide nebular area and therefore are not of CS origin but arise from within the nebula.

\section{acknowledgements}

This paper is a result of a project introduced by the Deanship of Scientific Research (DSR) at King Abdulaziz University, Jeddah, under grant no.(G-662-13037). The authors, therefore, acknowledge with thanks DSR for technical and financial support. The authors thank the anonymous referee for his/her valuable comments.

\section{REFERENCES}

Akras S., Gonçalves D. R., 2016, MNRAS, 455, 930

Ali A., Amer M. A., Dopita M. A., Vogt F. P. A., Basurah H. M., 2015, A\&A, 583, A83

Ali A., Dopita M. A., Basurah H. M., Amer M. A., Alsulami R., Alruhaili A., 2016, MNRAS, 462, 1393

Balick B., Preston H. L., Icke V., 1987, AJ, 94, 1641

Balick B., Rugers M., Terzian Y., Chengalur J. N., 1993, ApJ, 411, 778

Balick B., Perinotto M., Maccioni A., Terzian Y., Hajian A., 1994, ApJ, 424, 800

PASA (2018)

doi:10.1017/pas.2018.xxx
Balick B., Alexander J., Hajian A. R., Terzian Y., Perinotto M., Patriarchi P., 1998, AJ, 116, 360

Basurah H. M., Ali A., Dopita M. A., Alsulami R., Amer M. A., Alruhaili A., 2016, MNRAS,

Bond H. E., Schaefer K. G., Fullton L. K., Ciardullo R., 1995, in Butler B. J., Muhleman D. O., eds, BAASVol. 27, Bulletin of the American Astronomical Society. p. 1401

Cahn J. H., Kaler J. B., Stanghellini L., 1992, A\&AS, 94,399

Childress M. J., Vogt F. P. A., Nielsen J., Sharp R. G., 2014, Ap\&SS, 349, 617

Corradi R. L. M., Manso R., Mampaso A., Schwarz H. E., 1996, A\&A, 313, 913

Corradi R. L. M., Gonçalves D. R., Villaver E., Mampaso A., Perinotto M., 2000, ApJ, 542, 861

Costa R. D. D., Chiappini C., Maciel W. J., de Freitas Pacheco J. A., 1996, A\&AS, 116, 249

Delgado-Inglada G., Morisset C., Stasińska G., 2014, MNRAS, 440, 536

Dopita M. A., 1997, ApJ, 485, L41

Dopita M., Hart J., McGregor P., Oates P., Bloxham G., Jones D., 2007, Ap\&SS, 310, 255

Dopita M., et al., 2010, Ap\&SS, 327, 245

Dufour R. J., Kwitter K. B., Shaw R. A., Henry R. B. C., Balick B., Corradi R. L. M., 2015, ApJ, 803, 23

Durand S., Acker A., Zijlstra A., 1998, A\&AS, 132, 13

Dyson J. E., Redman M. P., 2000, in Kastner J. H., Soker N., Rappaport S., eds, Astronomical Society of the Pacific Conference Series Vol. 199, Asymmetrical Planetary Nebulae II: From Origins to Microstructures. p. 367

Frew D. J., Bojičić I. S., Parker Q. A., 2013, MNRAS, 431,2

García-Rojas J., Peña M., Peimbert A., 2009, A\&A, 496, 139

Gesicki K., Acker A., Zijlstra A. A., 2003, A\&A, 400,957

Gieseking F., Hippelein H., Weinberger R., 1986, A\&A, 156,101

Gonçalves D. R., 2004, in Meixner M., Kastner J. H., Balick B., Soker N., eds, Astronomical Society of the Pacific Conference Series Vol. 313, Asymmetrical Planetary Nebulae III: Winds, Structure and the Thunderbird. p. 216 (arXiv:astro-ph/0312527)

Gonçalves D. R., Corradi R. L. M., Mampaso A., 2001, ApJ, 547, 302

Górny S. K., 2014, A\&A, 570, A26

Gorny S. K., Stasińska G., Tylenda R., 1997, A\&A, 318,256

Grevesse N., Asplund M., Sauval A. J., Scott P., 2010, 
Ap\&SS, 328, 179

Gutierrez-Moreno A., Cortes G., Moreno H., 1985, PASP, 97, 397

Henry R. B. C., Balick B., Dufour R. J., Kwitter K. B., Shaw R. A., Miller T. R., Buell J. F., Corradi R. L. M., 2015, ApJ, 813, 121

Holovatyy V. V., Havrilova N. V., 2005, Astronomy Reports, 49, 390

Howarth I. D., 1983, MNRAS, 203, 301

Kaler J. B., Shaw R. A., Feibelman W. A., Imhoff C. L., 1991, PASP, 103, 67

Livio M., 1997, Space Sci. Rev., 82, 389

Martin W., 1981, A\&A, 98, 328

Milingo J. B., Kwitter K. B., Henry R. B. C., Cohen R. E., 2002a, ApJS, 138, 279

Milingo J. B., Henry R. B. C., Kwitter K. B., 2002b, ApJS, 138, 285

Milingo J. B., Kwitter K. B., Henry R. B. C., Souza S. P., 2010, ApJ, 711, 619

Miszalski B., 2009, PhD thesis, Department of Physics, Macquarie University, NSW 2109, Australia

Miszalski B., Corradi R. L. M., Boffin H. M. J., Jones D., Sabin L., Santander-García M., Rodríguez-Gil P., Rubio-Díez M. M., 2011, MNRAS, 413, 1264

Monreal-Ibero A., Roth M. M., Schönberner D., Steffen M., Böhm P., 2005, ApJ, 628, L139

Peimbert M., 1978, in Terzian Y., ed., IAU Symposium Vol. 76, Planetary Nebulae. pp 215-223

Pena M., Stasińska G., Esteban C., Koesterke L., Medina S., Kingsburgh R., 1998, A\&A, 337, 866

Perinotto M., 1991, ApJS, 76, 687

Perinotto M., Morbidelli L., Scatarzi A., 2004, MNRAS, 349, 793

Porter R. L., Ferland G. J., Storey P. J., Detisch M. J., 2012, MNRAS, 425, L28

Porter R. L., Ferland G. J., Storey P. J., Detisch M. J., 2013, MNRAS, 433, L89

Quireza C., Rocha-Pinto H. J., Maciel W. J., 2007, A\&A, 475, 217

Raga A. C., Riera A., Mellema G., Esquivel A., Velázquez P. F., 2008, A\&A, 489, 1141

Reid W. A., Parker Q. A., 2010, PASA, 27, 187

Ruiz M. T., Peimbert A., Peimbert M., Esteban C., 2003, ApJ, 595, 247

Sabbadin F., Minello S., Bianchini A., 1977, A\&A, 60,147

Steffen W., López J. A., Lim A. J., 2002, in Henney W. J., Steffen W., Binette L., Raga A., eds, Revista Mexicana de Astronomia y Astrofisica Conference Series Vol. 13, Revista Mexicana de Astronomia y Astrofisica Conference Series. pp 150-154
Storey P. J., Zeippen C. J., 2000, MNRAS, 312, 813

Tsamis Y. G., Walsh J. R., Pequignot D., Barlow M. J., Liu X.-W., Danziger I. J., 2007, The Messenger, 127,53

Tylenda R., Acker A., Stenholm B., Koeppen J., 1992, A\&AS, 95, 337

Weidmann W. A., Gamen R., 2011, A\&A, 526, A6

Wesson R., 2016, MNRAS, 456, 3774

Wesson R., Stock D. J., Scicluna P., 2012, MNRAS, 422,3516 


\section{Appendix: Emission Line Fluxes and ionic} abundances

The observed and de-reddened line fluxes (relative to $\mathrm{H} \beta$ $=100), \log \mathrm{F}(\mathrm{H} \beta)$, and $c(H \beta)$ of Hen 2-141, NGC 5307, IC 2553, and PB 6 as well as their studied knots were given in Tables A1 and A2. The [O III] line at $\lambda 5007$ is saturated in NGC 5307 and IC 2553 nebulae. The values of both lines were determined applying the theoretical line ratio $[\mathrm{O}$ III] $\lambda 5007$ / [O III] $\lambda 4959$ of 2.89 (Storey \& Zeippen 2000). Both of the [O III] lines at $\lambda 5007$ and $\lambda 4959$ are saturated in Hen 2-141, therefore we adopted their values as the averages of available values in literatures. Columns (1)and (2) give the laboratory wavelength and identification of observed lines, while other columns show the observed and de-reddened line fluxes of the PNe and their associated knots.

The total helium abundances for all objects were determined from $\mathrm{He}^{+} / \mathrm{H}$ and $\mathrm{He}^{2+} / \mathrm{H}$ ions using atomic data from Porter et al. (2012) and Porter et al. (2013). The total carbon abundances are determined from $\mathrm{C}^{2+} / \mathrm{H}$ and $\mathrm{C}^{3+} / \mathrm{H}$ ions in all objects, except Hen 2-141 which is determined from the $\mathrm{C}^{2+} / \mathrm{H}$ ion only. The ionic abundances of the four $\mathrm{PNe}$ and the knots were given in Tables A3 and A4. 
Table A1 Integrated line fluxes and de-reddened intensities, relative to $\mathrm{H} \beta=100$, of Hen 2-141 and NGC 5307.

\begin{tabular}{|c|c|c|c|c|c|c|c|c|c|c|c|}
\hline \multirow[b]{3}{*}{$\lambda_{\text {Lab }} \AA$} & \multirow[b]{3}{*}{ ID } & \multicolumn{2}{|c|}{ Hen 2-141 } & \multicolumn{8}{|c|}{ NGC 5307 } \\
\hline & & \multicolumn{2}{|c|}{ Entire nebula } & \multicolumn{2}{|c|}{ Entire nebula } & \multicolumn{2}{|c|}{$\mathrm{K}_{\mathrm{NW}}$} & \multicolumn{2}{|c|}{$\mathrm{K}_{\mathrm{SW}}$} & \multicolumn{2}{|c|}{$\mathrm{K}_{\mathrm{SE}}$} \\
\hline & & $\mathrm{F}(\lambda)$ & $\mathrm{I}(\lambda)$ & $F(\lambda)$ & $\mathrm{I}(\lambda)$ & $F(\lambda)$ & $\mathrm{I}(\lambda)$ & $F(\lambda)$ & $\mathrm{I}(\lambda)$ & $\mathrm{F}(\lambda)$ & $\mathrm{I}(\lambda)$ \\
\hline 3703.86 & H I & & & $1.75 \pm 0.14$ & $2.42 \pm 0.23$ & $1.96 \pm 0.32$ & $2.74 \pm 0.45$ & $1.13 \pm 0.17$ & $1.68 \pm 0.25$ & & \\
\hline 3711.97 & H I & $0.71 \pm 0.14$ & $1.20 \pm 0.23$ & $1.18 \pm 0.11$ & $1.63 \pm 0.16$ & & & $0.71 \pm 0.13$ & $1.06 \pm 0.20$ & & \\
\hline 3726.03 & {$[\mathrm{O}$ II] } & $46.83 \pm 6.14$ & $78.58 \pm 10.05$ & $10.03 \pm 0.25$ & $13.85 \pm 0.82$ & $81.16 \pm 3.82$ & $113.04 \pm 6.38$ & $15.52 \pm 0.41$ & $22.97 \pm 0.93$ & $29.13 \pm 4.17$ & $42.91 \pm 6.24$ \\
\hline 3728.82 & {$\left[\begin{array}{lll}\mathrm{O} & \mathrm{II}\end{array}\right]$} & $30.46 \pm 4.51$ & $50.91 \pm 7.27$ & $5.14 \pm 0.22$ & $7.08 \pm 0.47$ & $42.88 \pm 3.74$ & $59.35 \pm 5.30$ & $8.79 \pm 0.43$ & $12.98 \pm 0.73$ & $15.66 \pm 2.24$ & $23.02 \pm 3.36$ \\
\hline 3734.37 & H I & $1.00 \pm 0.21$ & $1.70 \pm 0.35$ & $1.68 \pm 0.08$ & $2.31 \pm 0.16$ & $2.27 \pm 0.48$ & $3.17 \pm 0.69$ & $1.57 \pm 0.19$ & $2.30 \pm 0.28$ & $0.87 \pm 0.26$ & $1.28 \pm 0.39$ \\
\hline 3750.15 & H I & $2.33 \pm 0.23$ & $3.89 \pm 0.37$ & $2.19 \pm 0.09$ & $3.00 \pm 0.20$ & $3.34 \pm 0.22$ & $4.62 \pm 0.32$ & $1.35 \pm 0.12$ & $1.99 \pm 0.18$ & $0.73 \pm 0.22$ & $1.07 \pm 0.32$ \\
\hline 3770.63 & H I & $2.70 \pm 0.24$ & $4.48 \pm 0.39$ & $2.85 \pm 0.13$ & $3.89 \pm 0.26$ & $4.43 \pm 0.29$ & $6.11 \pm 0.45$ & $2.88 \pm 0.17$ & $4.21 \pm 0.27$ & $1.22 \pm 0.25$ & $1.78 \pm 0.37$ \\
\hline 3797.90 & H I & $3.99 \pm 0.29$ & $6.57 \pm 0.49$ & $3.85 \pm 0.09$ & $5.24 \pm 0.30$ & $6.07 \pm 0.42$ & $8.29 \pm 0.60$ & $3.87 \pm 0.19$ & $5.62 \pm 0.32$ & $1.62 \pm 0.29$ & $2.34 \pm 0.40$ \\
\hline 3834.89 & He II & $1.31 \pm 0.32$ & $2.13 \pm 0.52$ & $5.36 \pm 0.09$ & $7.23 \pm 0.39$ & & & & & $1.13 \pm 0.33$ & $1.61 \pm 0.47$ \\
\hline 3868.75 & {$[\mathrm{Ne}$ III] } & $74.05 \pm 5.23$ & $118.41 \pm 8.45$ & $87.77 \pm 0.55$ & $117.37 \pm 5.83$ & $121.75 \pm 1.46$ & $163.77 \pm 4.87$ & $92.24 \pm 0.97$ & $131.15 \pm 3.88$ & $82.58 \pm 8.22$ & $116.08 \pm 11.27$ \\
\hline 3888.65 & He I & $10.37 \pm 0.67$ & $17.10 \pm 0.49$ & $14.65 \pm 0.12$ & $19.50 \pm 0.95$ & & & & & & \\
\hline 3967.46 & {$[\mathrm{Ne}$ III] } & $24.86 \pm 1.85$ & $38.15 \pm 2.85$ & $24.76 \pm 0.43$ & $32.29 \pm 1.56$ & $36.74 \pm 0.83$ & $48.19 \pm 1.63$ & $26.21 \pm 0.52$ & $36.15 \pm 1.16$ & $26.21 \pm 3.13$ & $35.66 \pm 4.12$ \\
\hline 3970.07 & H I & $9.99 \pm 1.54$ & $15.36 \pm 2.41$ & $11.27 \pm 0.41$ & $14.66 \pm 0.83$ & $18.81 \pm 0.69$ & $24.66 \pm 1.10$ & $11.95 \pm 0.50$ & $16.47 \pm 0.81$ & $10.69 \pm 0.55$ & $14.63 \pm 0.78$ \\
\hline 4068.60 & {$[\mathrm{~S} \mathrm{II}]$} & $1.70 \pm 0.10$ & $4.12 \pm 0.06$ & & & & & & & & \\
\hline 4100.04 & He II & $2.44 \pm 0.68$ & $3.53 \pm 0.96$ & & & & & & & & \\
\hline 4101.74 & H I & $19.11 \pm 0.68$ & $27.71 \pm 1.01$ & $20.82 \pm 0.10$ & $26.17 \pm 1.02$ & $30.57 \pm 0.29$ & $38.63 \pm 0.92$ & $21.16 \pm 0.23$ & $27.93 \pm 0.68$ & $17.83 \pm 1.37$ & $23.35 \pm 1.70$ \\
\hline 4267.15 & C II & $0.48 \pm 0.10$ & $0.65 \pm 0.14$ & $0.13 \pm 0.02$ & $0.15 \pm 0.03$ & & & $0.20 \pm 0.04$ & $0.25 \pm 0.05$ & & \\
\hline 4340.47 & H I & $36.93 \pm 0.30$ & $47.91 \pm 0.60$ & $39.71 \pm 0.31$ & $46.59 \pm 1.30$ & $54.46 \pm 0.91$ & $64.12 \pm 1.44$ & $40.87 \pm 1.05$ & $49.59 \pm 1.41$ & $38.43 \pm 2.30$ & $46.41 \pm 2.57$ \\
\hline 4363.21 & [O III] & $13.40 \pm 0.15$ & $17.19 \pm 0.25$ & $13.74 \pm 0.23$ & $16.01 \pm 0.49$ & $12.75 \pm 0.29$ & $14.91 \pm 0.40$ & $13.35 \pm 0.35$ & $16.07 \pm 0.48$ & $12.15 \pm 0.71$ & $14.56 \pm 0.84$ \\
\hline 4471.50 & He I & $2.11 \pm 0.12$ & $2.56 \pm 0.15$ & $3.47 \pm 0.03$ & $3.91 \pm 0.09$ & $1.17 \pm 0.20$ & $1.33 \pm 0.23$ & $4.15 \pm 0.07$ & $4.81 \pm 0.10$ & $4.32 \pm 0.31$ & $4.98 \pm 0.36$ \\
\hline 4541.59 & He II & $2.07 \pm 0.07$ & $2.43 \pm 0.09$ & $0.73 \pm 0.03$ & $0.80 \pm 0.03$ & & & & & & \\
\hline 4634.14 & N III & $0.71 \pm 0.09$ & $0.80 \pm 0.10$ & & & & & & & & \\
\hline 4640.64 & N III & $1.46 \pm 0.08$ & $1.63 \pm 0.09$ & $0.96 \pm 0.02$ & $1.03 \pm 0.02$ & & & $0.55 \pm 0.02$ & $0.60 \pm 0.02$ & $0.55 \pm 0.03$ & $0.60 \pm 0.03$ \\
\hline 4641.81 & O II & $0.28 \pm 0.08$ & $0.51 \pm 0.00$ & $0.18 \pm 0.01$ & $0.20 \pm 0.01$ & & & & & & \\
\hline 4649.13 & O II & $0.28 \pm 0.08$ & $0.31 \pm 0.08$ & $0.14 \pm 0.02$ & $0.15 \pm 0.02$ & & & & & & \\
\hline 4650.25 & C III & & & $0.15 \pm 0.02$ & $0.17 \pm 0.02$ & $0.13 \pm 0.03$ & $0.14 \pm 0.04$ & $0.15 \pm 0.03$ & $0.16 \pm 0.03$ & & \\
\hline 4685.68 & He II & $63.61 \pm 0.52$ & $69.50 \pm 0.62$ & $23.03 \pm 0.19$ & $24.32 \pm 0.30$ & $1.41 \pm 0.10$ & $1.49 \pm 0.11$ & $7.34 \pm 0.19$ & $7.84 \pm 0.20$ & $1.53 \pm 0.09$ & $1.63 \pm 0.10$ \\
\hline 4711.37 & {$[\mathrm{Ar} I V]$} & $7.28 \pm 0.17$ & $7.85 \pm 0.19$ & $4.79 \pm 0.10$ & $5.02 \pm 0.12$ & $2.12 \pm 0.11$ & $2.22 \pm 0.11$ & $3.39 \pm 0.09$ & $3.59 \pm 0.09$ & $2.95 \pm 0.17$ & $3.11 \pm 0.18$ \\
\hline 4724.89 & {$[\mathrm{Ne}$ IV] } & $1.73 \pm 0.10$ & $1.86 \pm 0.11$ & & & & & & & & \\
\hline 4740.17 & {$[\mathrm{Ar}$ IV $]$} & $6.26 \pm 0.10$ & $6.66 \pm 0.11$ & $4.14 \pm 0.07$ & $4.30 \pm 0.07$ & $1.84 \pm 0.10$ & $1.91 \pm 0.10$ & $3.02 \pm 0.09$ & $3.16 \pm 0.09$ & $2.60 \pm 0.14$ & $2.72 \pm 0.15$ \\
\hline 4861.33 & H I & $100.00 \pm 1.64$ & $100.00 \pm 1.64$ & $100.00 \pm 2.94$ & $100.00 \pm 2.94$ & $100.00 \pm 4.44$ & $100.00 \pm 4.46$ & $100.00 \pm 6.19$ & $100.00 \pm 6.13$ & $100.00 \pm 2.37$ & $100.00 \pm 2.36$ \\
\hline 4921.93 & He I & $0.64 \pm 0.14$ & $0.62 \pm 0.14$ & $1.13 \pm 0.04$ & $1.11 \pm 0.04$ & $1.81 \pm 0.08$ & $1.78 \pm 0.08$ & $1.23 \pm 0.10$ & $1.20 \pm 0.09$ & $1.24 \pm 0.05$ & $1.22 \pm 0.05$ \\
\hline 4958.91 & [O III] & $519.98 \pm 1.65$ & $495.00 \pm 1.85$ & $459.97 \pm 42.79$ & $444.00 \pm 40.39$ & $426.78 \pm 18.09$ & $414.00 \pm 17.70$ & $469.92 \pm 35.11$ & $453.00 \pm 33.89$ & $452.25 \pm 36.98$ & $436.00 \pm 35.64$ \\
\hline 5006.84 & [O III] & $1606.35 \pm 23.19$ & $1493.00 \pm 22.00$ & $1402.29 \pm 134.23$ & $1336.00 \pm 128.00$ & $1268.20 \pm 46.90$ & $1211.00 \pm 45.00$ & $1503.03 \pm 22.86$ & $1464.00 \pm 22.00$ & $1249.39 \pm 52.73$ & $1184.00 \pm 50.00$ \\
\hline 5015.68 & He I & $3.37 \pm 0.14$ & $3.12 \pm 0.13$ & $1.83 \pm 0.33$ & $1.75 \pm 0.32$ & $1.23 \pm 0.32$ & $1.17 \pm 0.30$ & & & $2.18 \pm 0.53$ & $2.05 \pm 0.50$ \\
\hline 5199.84 & {$[\mathrm{~N} \mathrm{I}]$} & $2.31 \pm 0.33$ & $3.20 \pm 0.02$ & $0.10 \pm 0.03$ & $0.09 \pm 0.03$ & & & $0.15 \pm 0.01$ & $0.13 \pm 0.11$ & $0.24 \pm 0.02$ & $0.22 \pm 0.02$ \\
\hline 5200.26 & {$[\mathrm{~N} \mathrm{I}]$} & & & $0.08 \pm 0.03$ & $0.07 \pm 0.03$ & $0.50 \pm 0.14$ & $0.45 \pm 0.12$ & $0.08 \pm 0.01$ & $0.07 \pm 0.07$ & $0.30 \pm 0.02$ & $0.26 \pm 0.02$ \\
\hline 5411.52 & He II & $7.10 \pm 0.39$ & $5.40 \pm 0.30$ & $2.28 \pm 0.21$ & $1.92 \pm 0.18$ & & & $0.82 \pm 0.07$ & $0.66 \pm 0.05$ & $0.19 \pm 0.04$ & $0.16 \pm 0.03$ \\
\hline 5517.66 & [Cl III] & $1.33 \pm 0.29$ & $0.97 \pm 0.22$ & $0.87 \pm 0.07$ & $0.71 \pm 0.06$ & $1.55 \pm 0.51$ & $1.28 \pm 0.41$ & $0.58 \pm 0.05$ & $0.46 \pm 0.04$ & $0.89 \pm 0.24$ & $0.71 \pm 0.19$ \\
\hline 5537.60 & {$[\mathrm{Cl} \mathrm{III}]$} & $1.18 \pm 0.23$ & $0.86 \pm 0.16$ & $0.80 \pm 0.06$ & $0.65 \pm 0.05$ & $1.42 \pm 0.54$ & $1.15 \pm 0.43$ & $0.60 \pm 0.07$ & $0.47 \pm 0.06$ & $0.86 \pm 0.20$ & $0.68 \pm 0.16$ \\
\hline 5754.60 & {$[\mathrm{~N}$ II] } & $3.28 \pm 0.32$ & $2.20 \pm 0.21$ & $0.38 \pm 0.05$ & $0.30 \pm 0.04$ & $3.41 \pm 0.14$ & $2.66 \pm 0.12$ & $1.05 \pm 0.05$ & $0.78 \pm 0.04$ & $1.51 \pm 0.05$ & $1.13 \pm 0.04$ \\
\hline 5801.51 & C IV & $0.94 \pm 0.19$ & $0.62 \pm 0.13$ & $0.14 \pm 0.03$ & $0.11 \pm 0.02$ & & & & & & \\
\hline 5812.14 & C IV & & & $0.08 \pm 0.02$ & $0.07 \pm 0.01$ & & & $0.10 \pm 0.03$ & $0.08 \pm 0.02$ & & \\
\hline 5875.66 & He I & $12.93 \pm 0.50$ & $8.33 \pm 0.35$ & $16.07 \pm 1.85$ & $12.20 \pm 1.45$ & $23.90 \pm 0.19$ & $18.14 \pm 0.49$ & $26.78 \pm 0.14$ & $19.31 \pm 0.51$ & $19.39 \pm 0.06$ & $14.06 \pm 0.20$ \\
\hline 6300.34 & {$[\mathrm{O}$} & $99 \pm 1.11$ & $7.83 \pm 0.62$ & $1 \pm 0.22$ & $1.47 \pm 0.17$ & $19.91 \pm 1.00$ & $13.84 \pm 0.81$ & $6.90 \pm 0.51$ & $4.48 \pm 0.35$ & $8.44 \pm 0.26$ & $5.54 \pm 0.20$ \\
\hline 6312.10 & [S III] & $4.41 \pm 0.20$ & $2.46 \pm 0.12$ & $1.73 \pm 0.13$ & $1.20 \pm 0.11$ & $6.32 \pm 0.44$ & $4.38 \pm 0.32$ & $2.82 \pm 0.14$ & $1.83 \pm 0.11$ & $3.14 \pm 0.24$ & $2.06 \pm 0.16$ \\
\hline 6363.78 & {$\left[\begin{array}{ll}\mathrm{O} & \mathrm{I}\end{array}\right]$} & $4.74 \pm 0.40$ & $2.60 \pm 0.22$ & $0.79 \pm 0.09$ & $0.54 \pm 0.07$ & $7.28 \pm 0.25$ & $5.01 \pm 0.24$ & $2.32 \pm 0.17$ & $1.48 \pm 0.12$ & $2.48 \pm 0.13$ & $1.60 \pm 0.09$ \\
\hline 6548.10 & {$[\mathrm{~N}$ II] } & $72.19 \pm 3.73$ & $37.52 \pm 2.12$ & $5.02 \pm 0.44$ & $3.36 \pm 0.35$ & $46.61 \pm 3.33$ & $30.87 \pm 2.42$ & $12.60 \pm 0.82$ & $7.73 \pm 0.56$ & $21.29 \pm 2.25$ & $13.14 \pm 1.37$ \\
\hline 6562.77 & H I & $548.05 \pm 13.47$ & $284.00 \pm 3.40$ & $421.82 \pm 31.84$ & $281.00 \pm 5.60$ & $458.98 \pm 3.46$ & $304.00 \pm 11.60$ & $467.07 \pm 1.50$ & $287.00 \pm 11.10$ & $450.30 \pm 2.20$ & $279.00 \pm 5.50$ \\
\hline 6583.50 & {$[\mathrm{~N}$ II $]$} & $216.38 \pm 21.24$ & $111.10 \pm 1$ & $14.67 \pm 1.59$ & $9.72 \pm 1.19$ & $144.46 \pm 13.78$ & $94.84 \pm 9.45$ & $40.94 \pm 1.80$ & $25.02 \pm 1.47$ & $66.38 \pm 5.13$ & $40.74 \pm 3.14$ \\
\hline 6678.16 & He I & $3.98 \pm 0.13$ & $1.99 \pm 0.08$ & $4.94 \pm 0.32$ & $3.23 \pm 0.30$ & $10.02 \pm 0.12$ & $6.50 \pm 0.27$ & $7.86 \pm 0.51$ & $4.70 \pm 0.35$ & $6.18 \pm 0.27$ & $3.74 \pm 0.18$ \\
\hline 6716.44 & [S II] & $11.66 \pm 0.25$ & $5.78 \pm 0$. & $2.07 \pm 0.14$ & $1.35 \pm 0.13$ & $20.86 \pm 0.90$ & $13.43 \pm 0.77$ & $4.71 \pm 0.20$ & $2.80 \pm 0.16$ & $8.16 \pm 0.32$ & $4.89 \pm 0.22$ \\
\hline 6730.82 & {$\left[\begin{array}{lll}\mathrm{S} & \mathrm{II}\end{array}\right]$} & $15.35 \pm 0.36$ & $7.58 \pm 0.27$ & $3.49 \pm 0.25$ & $2.26 \pm 0.22$ & $28.76 \pm 1.26$ & $18.47 \pm 1.07$ & $7.63 \pm 0.47$ & $4.50 \pm 0.32$ & $13.11 \pm 0.60$ & $7.83 \pm 0.39$ \\
\hline 6890.88 & He I & $2.06 \pm 0.19$ & $0.97 \pm 0.09$ & $0.46 \pm 0.04$ & $0.29 \pm 0.03$ & & & & & & \\
\hline 7005.67 & {$[\mathrm{Ar} \mathrm{V}]$} & $2.94 \pm 0.24$ & $1.35 \pm 0.11$ & $0.37 \pm 0.03$ & $0.23 \pm 0.03$ & & & & & & \\
\hline$c(H \beta)$ & & & & & & & & & & & \\
\hline $\mathrm{F}(H \beta)$ & & & .78 & -11 & & & & & & & 78 \\
\hline
\end{tabular}


Table A2 Integrated line fluxes and de-reddened intensities, relative to $\mathrm{H} \beta=100$, of IC 2553 and PB6.

\begin{tabular}{|c|c|c|c|c|c|c|c|c|c|c|c|}
\hline \multirow{4}{*}{$\lambda_{\text {Lab }} \AA$} & \multirow[b]{3}{*}{ ID } & \multicolumn{6}{|c|}{ IC 2553} & \multicolumn{4}{|c|}{$\mathrm{PB} 6$} \\
\hline & & \multicolumn{2}{|c|}{ Entire nebula } & \multicolumn{2}{|c|}{$\mathrm{K}_{\mathrm{NE}}$} & \multicolumn{2}{|c|}{$\mathrm{K}_{\mathrm{SW}}$} & \multicolumn{2}{|c|}{ Entire nebula } & \multirow{2}{*}{\multicolumn{2}{|c|}{$\mathrm{K}_{\mathrm{SE}}$}} \\
\hline & & $F(\lambda)$ & $I(\lambda)$ & $F(\lambda)$ & $\mathrm{I}(\lambda)$ & $F(\lambda)$ & $\mathrm{I}(\lambda)$ & $F(\lambda)$ & $\mathrm{I}(\lambda)$ & & \\
\hline & H I & $1.68 \pm 0.29$ & $2.16 \pm 0.38$ & & & & & $1.08 \pm 0.22$ & $1.55 \pm 0.32$ & & \\
\hline 3726.03 & [O II] & $14.50 \pm 1.36$ & $18.47 \pm 1.88$ & $43.41 \pm 3.91$ & $58.14 \pm 5.19$ & $54.86 \pm 4.94$ & $70.65 \pm 6.29$ & $30.76 \pm 1.18$ & $44.13 \pm 1.86$ & $56.38 \pm 5.65$ & $75.20 \pm 7.54$ \\
\hline 3728.82 & {$\left[\begin{array}{lll}\mathrm{O} & \mathrm{II}\end{array}\right]$} & $6.96 \pm 1.24$ & $8.88 \pm 1.59$ & $22.20 \pm 1.89$ & $29.68 \pm 2.52$ & $29.94 \pm 2.69$ & $38.80 \pm 3.65$ & $19.36 \pm 1.18$ & $27.78 \pm 1.76$ & $36.57 \pm 3.29$ & $49.07 \pm 4.52$ \\
\hline 3734.37 & H I & $2.11 \pm 0.22$ & $2.70 \pm 0.29$ & & & & $3.16 \pm 0.28$ & $1.72 \pm 0.19$ & $2.45 \pm 0.27$ & & \\
\hline 3750.15 & H I & $2.58 \pm 0.22$ & $3.29 \pm 0.30$ & $3.99 \pm 0.36$ & $5.32 \pm 0.48$ & $3.61 \pm 0.33$ & $4.63 \pm 0.42$ & $1.45 \pm 0.21$ & $2.07 \pm 0.31$ & & \\
\hline 3770.63 & H I & $3.24 \pm 0.24$ & $4.11 \pm 0.35$ & $3.84 \pm 0.35$ & $5.10 \pm 0.46$ & $3.80 \pm 0.34$ & $4.86 \pm 0.43$ & $2.60 \pm 0.22$ & $3.69 \pm 0.31$ & $3.58 \pm 0.32$ & $4.74 \pm 0.42$ \\
\hline 3797.90 & H I & $4.44 \pm 0.28$ & $5.61 \pm 0.42$ & $5.62 \pm 0.51$ & $7.41 \pm 0.66$ & $5.09 \pm 0.46$ & $6.47 \pm 0.58$ & $5.20 \pm 0.63$ & $7.34 \pm 0.89$ & $3.83 \pm 0.34$ & $5.04 \pm 0.45$ \\
\hline 3834.89 & He II & $6.33 \pm 0.24$ & $7.95 \pm 0.46$ & $7.44 \pm 0.67$ & $9.74 \pm 0.87$ & & & $6.45 \pm 0.68$ & $10.54 \pm 0.16$ & $6.00 \pm 0.54$ & $7.84 \pm 0.70$ \\
\hline 3868.75 & [Ne III] & $86.05 \pm 3.47$ & $107.35 \pm 6.23$ & $114.33 \pm 10.98$ & $148.26 \pm 13.90$ & $125.68 \pm 6.28$ & $158.26 \pm 8.59$ & $85.91 \pm 1.11$ & $118.84 \pm 2.38$ & $101.99 \pm 5.10$ & $132.94 \pm 7.14$ \\
\hline 3888.65 & He I & $16.65 \pm 0.49$ & $20.72 \pm 1.06$ & $20.06 \pm 1.81$ & $25.93 \pm 2.31$ & $20.72 \pm 1.86$ & $25.92 \pm 2.33$ & $13.01 \pm 0.41$ & $20.97 \pm 0.31$ & $15.54 \pm 1.40$ & $20.09 \pm 1.78$ \\
\hline 3967.46 & [Ne III] & $32.18 \pm 0.79$ & $39.45 \pm 1.87$ & $37.77 \pm 2.27$ & $47.98 \pm 2.93$ & $39.38 \pm 3.54$ & $48.44 \pm 4.34$ & $22.15 \pm 0.33$ & $29.80 \pm 0.60$ & $23.59 \pm 2.12$ & $29.94 \pm 2.68$ \\
\hline 3970.07 & $\mathrm{H} \mathrm{I}$ & $12.41 \pm 0.68$ & $15.17 \pm 1.01$ & $13.97 \pm 1.26$ & $17.69 \pm 1.56$ & $15.28 \pm 1.38$ & $18.82 \pm 1.67$ & $12.06 \pm 0.30$ & $16.21 \pm 0.46$ & $12.35 \pm 1.11$ & $15.73 \pm 1.45$ \\
\hline 4026.08 & N II & $2.01 \pm 0.04$ & $2.44 \pm 0.10$ & $2.35 \pm 0.21$ & $2.94 \pm 0.26$ & $2.58 \pm 0.23$ & $3.14 \pm 0.28$ & $1.96 \pm 0.23$ & $3.03 \pm 0.04$ & & \\
\hline 4068.60 & [S II] & $1.60 \pm 0.04$ & $1.93 \pm 0.09$ & $3.44 \pm 0.31$ & $4.28 \pm 0.39$ & $4.53 \pm 0.41$ & $5.48 \pm 0.51$ & & & $2.99 \pm 0.27$ & $3.69 \pm 0.33$ \\
\hline 4076.35 & [S II] & $0.71 \pm 0.03$ & $0.85 \pm 0.05$ & 1.250 .11 & & $1.75 \pm 0.16$ & $2.10 \pm 0.19$ & & & & \\
\hline 4100.04 & He II & $0.29 \pm 0.08$ & $0.34 \pm 0.10$ & & & & & $1.67 \pm 0.20$ & $2.15 \pm 0.26$ & & \\
\hline 4101.74 & H I & $22.09 \pm 0.30$ & $26.34 \pm 0.98$ & $25.07 \pm 1.50$ & $30.88 \pm 1.86$ & $25.73 \pm 1.29$ & $30.86 \pm 1.55$ & $21.10 \pm 0.23$ & $27.25 \pm 0.43$ & $22.48 \pm 1.12$ & $27.69 \pm 1.38$ \\
\hline 4199.83 & He II & $0.47 \pm 0.06$ & $0.55 \pm 0.08$ & & & & & $2.29 \pm 0.08$ & $3.36 \pm 0.04$ & $1.52 \pm 0.14$ & $1.82 \pm 0.16$ \\
\hline 4267.15 & C II & $0.54 \pm 0.01$ & $0.63 \pm 0.02$ & $0.52 \pm 0.10$ & $0.62 \pm 0.12$ & $0.48 \pm 0.10$ & $0.56 \pm 0.11$ & $0.36 \pm 0.08$ & $0.44 \pm 0.09$ & & \\
\hline 4340.47 & H I & $40.87 \pm 0.40$ & $46.21 \pm 1.20$ & $37.68 \pm 2.26$ & $43.57 \pm 2.45$ & $46.53 \pm 2.33$ & $52.82 \pm 2.55$ & $38.98 \pm 0.44$ & $46.60 \pm 0.62$ & $40.46 \pm 2.02$ & $46.85 \pm 2.18$ \\
\hline 4363.21 & {$[\mathrm{O} \mathrm{III}]$} & $9.30 \pm 0.11$ & $10.46 \pm 0.27$ & $8.61 \pm 0.77$ & $9.85 \pm 0.85$ & $9.28 \pm 0.84$ & $10.42 \pm 0.92$ & $16.62 \pm 0.32$ & $19.72 \pm 0.41$ & $15.73 \pm 1.42$ & $18.01 \pm 1.60$ \\
\hline 4471.50 & He I & $4.22 \pm 0.04$ & $4.63 \pm 0.09$ & $4.96 \pm 0.45$ & $5.52 \pm 0.48$ & $5.35 \pm 0.48$ & $5.89 \pm 0.53$ & $2.03 \pm 0.09$ & $2.33 \pm 0.11$ & $3.68 \pm 0.33$ & $4.09 \pm 0.36$ \\
\hline 4541.59 & He II & $0.78 \pm 0.03$ & $0.85 \pm 0.03$ & 0.650 .06 & & & & $4.38 \pm 0.09$ & $4.89 \pm 0.11$ & $3.77 \pm 0.34$ & $4.12 \pm 0.37$ \\
\hline 4634.14 & N III & $1.77 \pm 0.08$ & $1.87 \pm 0.09$ & $1.10 \pm 0.10$ & $1.17 \pm 0.10$ & $0.61 \pm 0.05$ & $0.64 \pm 0.06$ & $1.04 \pm 0.14$ & $1.12 \pm 0.14$ & & \\
\hline 4640.64 & N III & $3.64 \pm 0.05$ & $3.83 \pm 0.06$ & $2.62 \pm 0.24$ & $2.78 \pm 0.24$ & $2.05 \pm 0.18$ & $2.16 \pm 0.20$ & $1.42 \pm 0.08$ & $1.54 \pm 0.09$ & & \\
\hline 4647.42 & C III & & & & & & & $0.45 \pm 0.07$ & $0.49 \pm 0.08$ & & \\
\hline 4650.25 & C III & $0.92 \pm 0.06$ & $0.96 \pm 0.06$ & $0.83 \pm 0.07$ & $0.88 \pm 0.08$ & & & & & & \\
\hline 4658.64 & C IV & $0.20 \pm 0.03$ & $0.21 \pm 0.03$ & & & & & & & & \\
\hline 4685.68 & He II & $24.14 \pm 0.38$ & $25.17 \pm 0.45$ & $14.51 \pm 1.31$ & $15.23 \pm 1.37$ & $9.49 \pm 0.28$ & $9.91 \pm 0.30$ & $129.07 \pm 0.77$ & $137.17 \pm 0.91$ & $96.84 \pm 2.91$ & $101.76 \pm 3.06$ \\
\hline 4711.37 & {$[\mathrm{Ar}$ IV] } & $5.39 \pm 0.07$ & $5.59 \pm 0.08$ & $2.82 \pm 0.25$ & $2.95 \pm 0.26$ & $2.55 \pm 0.23$ & $2.66 \pm 0.24$ & $9.76 \pm 0.22$ & $10.28 \pm 0.23$ & $7.16 \pm 0.72$ & $7.44 \pm 0.72$ \\
\hline 4715.21 & [Ne IV] & & & & & & & $1.62 \pm 0.20$ & $1.70 \pm 0.20$ & & \\
\hline 4724.15 & {$[\mathrm{Ne}$ IV] } & 0.280 .04 & & & & & & $1.65 \pm 0.08$ & $1.74 \pm 0.09$ & & \\
\hline 4725.62 & [Ne IV] & & & & & & & $1.34 \pm 0.07$ & $1.40 \pm 0.07$ & & \\
\hline 4740.17 & [Ar IV] & $6.03 \pm 0.11$ & $6.21 \pm 0.12$ & $2.91 \pm 0.26$ & $3.02 \pm 0.27$ & $2.63 \pm 0.28$ & $2.71 \pm 0.29$ & $8.85 \pm 0.18$ & $9.23 \pm 0.18$ & $6.31 \pm 0.57$ & $6.50 \pm 0.56$ \\
\hline 4859.32 & He II & & & & & & & $6.76 \pm 0.85$ & $6.78 \pm 0.85$ & & \\
\hline 4861.33 & H I & $100.00 \pm 3.82$ & $100.00 \pm 3.86$ & $100.00 \pm 3.00$ & $100.00 \pm 2.98$ & $100.00 \pm 3.00$ & $100.00 \pm 2.96$ & $100.00 \pm 0.86$ & $100.00 \pm 0.85$ & $100.00 \pm 3.00$ & $100.00 \pm 3.04$ \\
\hline 4958.91 & [O III] & $444.84 \pm 16.22$ & $434.39 \pm 15.79$ & $419.56 \pm 37.76$ & $406.66 \pm 35.66$ & $412.84 \pm 12.39$ & $403.07 \pm 1$ & $365.24 \pm 2.12$ & $353.13 \pm 2.13$ & $379.38 \pm 11.38$ & $369.00 \pm 11.20$ \\
\hline 5006.84 & {$\left[\begin{array}{lll}\mathrm{O} & \mathrm{III}\end{array}\right]$} & $1289.98 \pm 46.98$ & $1245.55 \pm 45.94$ & $1220.10 \pm 36.60$ & $1170.78 \pm 35.42$ & $1240.62 \pm 37.22$ & $1197.60 \pm 36.13$ & $1098.89 \pm 4.35$ & $1045.01 \pm 4.78$ & $1199.83 \pm 35.99$ & $1151.54 \pm 34.37$ \\
\hline 5191.82 & [Ar III] & $0.14 \pm 0.01$ & $0.13 \pm 0.01$ & $0.16 \pm 0.01$ & $0.15 \pm 0.01$ & & & & & & \\
\hline 5197.81 & {$[\mathrm{~N} \mathrm{I}]$} & $0.14 \pm 0.01$ & $0.13 \pm 0.01$ & $0.28 \pm 0.03$ & $0.25 \pm 0.02$ & $0.72 \pm 0.07$ & $0.66 \pm 0.06$ & $1.20 \pm 0.34$ & $1.07 \pm 0.31$ & 3.070 .28 & \\
\hline 5200.26 & {$[\mathrm{~N} \mathrm{I}]$} & $0.10 \pm 0.03$ & $0.09 \pm 0.02$ & $0.22 \pm 0.02$ & $0.20 \pm 0.02$ & $0.62 \pm 0.06$ & $0.57 \pm 0.05$ & $1.03 \pm 0.34$ & $0.92 \pm 0.31$ & 1.980 .18 & \\
\hline 5411.52 & He II & $2.16 \pm 0.13$ & $1.90 \pm 0.12$ & $1.19 \pm 0.11$ & $1.02 \pm 0.09$ & $050+005$ & $0.51 \pm 0.05$ & $14.02 \pm 0.95$ & $11.63 \pm 0.79$ & $9.47 \pm 0.85$ & 8.1 \\
\hline 5517.66 & {$[\mathrm{Cl} \mathrm{III}]$} & $0.77 \pm 0.03$ & $0.66 \pm 0.03$ & $0.78 \pm 0.07$ & $0.65 \pm 0.06$ & $0.89 \pm 0.08$ & $0.76 \pm 0.07$ & $1.05 \pm 0.22$ & $0.85 \pm 0.18$ & $0.74 \pm 0.06$ & $0.62 \pm 0.05$ \\
\hline 5537.60 & [Cl III] & $0.99 \pm 0.09$ & $0.84 \pm 0.08$ & $0.91 \pm 0.08$ & $0.76 \pm 0.07$ & $0.93 \pm 0.07$ & $0.79 \pm 0.06$ & $1.04 \pm 0.29$ & $0.83 \pm 0.23$ & $0.73 \pm 0.10$ & $0.61 \pm 0.08$ \\
\hline 5592.37 & O III & $0.09 \pm 0.01$ & $0.08 \pm 0.01$ & & & & & & & & \\
\hline 5754.60 & {$[\mathrm{~N} \mathrm{II}]$} & $0.90 \pm 0.09$ & $0.74 \pm 0.08$ & $2.43 \pm 0.15$ & $1.94 \pm 0.12$ & $2.36 \pm 0.21$ & $1.93 \pm 0.17$ & $6.63 \pm 0.42$ & $5.03 \pm 0.32$ & $9.27 \pm 0.75$ & $7.39 \pm 0.59$ \\
\hline 5801.51 & C IV & $0.08 \pm 0.01$ & $0.07 \pm 0.01$ & & & & & $0.76 \pm 0.13$ & $0.57 \pm 0.10$ & & \\
\hline 5812.14 & C IV & $0.05 \pm 0.01$ & $0.04 \pm 0.01$ & & & & & $0.53 \pm 0.14$ & $0.40 \pm 0.10$ & & \\
\hline 5875.66 & $\mathrm{He} \mathrm{I}$ & $16.88 \pm 1.51$ & $13.66 \pm 1.29$ & $12.12 \pm 1.09$ & $9.44 \pm 0.85$ & $17.53 \pm 1.58$ & $14.08 \pm 1.25$ & $10.73 \pm 0.57$ & $7.92 \pm 0.44$ & $12.39 \pm 1.12$ & $9.64 \pm 0.86$ \\
\hline 6300.34 & {$[\mathrm{O} \mathrm{I}]$} & $1.86 \pm 0.10$ & $1.41 \pm 0.11$ & $4.70 \pm 0.42$ & $3.39 \pm 0.30$ & $5.81 \pm 0.52$ & $4.37 \pm 0.40$ & $6.63 \pm 0.18$ & $4.46 \pm 0.15$ & $9.46 \pm 0.85$ & $6.82 \pm 0.62$ \\
\hline 6312.10 & [S III] & $3.05 \pm 0.21$ & $2.31 \pm 0.20$ & $2.49 \pm 0.22$ & $1.79 \pm 0.16$ & $3.86 \pm 0.35$ & $2.90 \pm 0.28$ & $4.78 \pm 0.14$ & $3.21 \pm 0.11$ & $3.39 \pm 0.31$ & $2.44 \pm 0.22$ \\
\hline 6363.78 & {$[\mathrm{O} I]$} & $0.61 \pm 0.04$ & $0.46 \pm 0.04$ & $1.61 \pm 0.05$ & $1.16 \pm 0.05$ & $2.04 \pm 0.18$ & $1.52 \pm 0.14$ & $2.29 \pm 0.07$ & $1.52 \pm 0.05$ & $3.47 \pm 0.31$ & $2.47 \pm 0.22$ \\
\hline 6548.10 & [N II] & $17.18 \pm 1.14$ & $12.61 \pm 1.09$ & $51.28 \pm 0.95$ & $35.61 \pm 1.16$ & $44.33 \pm 1.33$ & $32.23 \pm 1.42$ & $119.83 \pm 4.09$ & $76.58 \pm 3.02$ & $2 \pm 5.54$ & $128.17 \pm 5.31$ \\
\hline 6560.10 & He II & & & & & & & $28.54 \pm 7.49$ & $18.11 \pm 4.71$ & $13.30 \pm 1.20$ & $9.16 \pm 0.82$ \\
\hline 6562.77 & H I & $386.61 \pm 25.75$ & $283.14 \pm 6.50$ & $410.39 \pm 7.15$ & $284.34 \pm 6.38$ & $409.33 \pm 11.24$ & $296.69 \pm 6.61$ & $438.20 \pm 9.86$ & $279.12 \pm 1.75$ & $406.06 \pm 8.98$ & $281.26 \pm 6.03$ \\
\hline 6583.50 & {$[\mathrm{~N} \mathrm{II}]$} & $46.16 \pm 3.24$ & $33.69 \pm 3.03$ & $143.64 \pm 4.86$ & $99.18 \pm 4.28$ & $142.17 \pm 4.27$ & $102.82 \pm 4.54$ & $340.81 \pm 8.30$ & $216.47 \pm 7.01$ & $534.80 \pm 1.00$ & $369.29 \pm 10.71$ \\
\hline 6678.16 & He I & $5.08 \pm 0.30$ & $3.66 \pm 0.31$ & $3.35 \pm 0.30$ & $2.27 \pm 0.21$ & $6.71 \pm 0.34$ & $4.78 \pm$ & $3.23 \pm 0.13$ & $2.01 \pm 0.09$ & $4.36 \pm 0.39$ & $2.95 \pm 0.27$ \\
\hline .44 & [S II] & $4.28 \pm 0.25$ & $3.08 \pm 0.26$ & $9.07 \pm 0.54$ & $6.12 \pm 0.40$ & \pm 0.69 & \pm 0.58 & $10.10 \pm 0.2$ & $6.24 \pm 0.21$ & $13.97 \pm 0.70$ & $9.43 \pm 0.54$ \\
\hline 6730.82 & [S II] & $7.08 \pm 0.38$ & $5.07 \pm 0.42$ & $14.89 \pm 0.89$ & $10.03 \pm 0.66$ & $19.50 \pm 1.76$ & $13.76 \pm 1.27$ & $14.22 \pm 0.38$ & $8.77 \pm 0.31$ & $18.79 \pm 0.97$ & $12.67 \pm 0.76$ \\
\hline 6890.88 & He I & $0.33 \pm 0.03$ & $0.23 \pm 0.03$ & & & & & $2.18 \pm 0.09$ & $1.30 \pm 0.06$ & & \\
\hline 7005.67 & {$[\mathrm{Ar} \mathrm{V}]$} & $0.83 \pm 0.09$ & $0.57 \pm 0.07$ & & & & & $7.00 \pm 0.18$ & $4.10 \pm 0.14$ & $2.08 \pm 0.19$ & $1.34 \pm 0.12$ \\
\hline ( & & & & & & & & & & & \\
\hline $\mathrm{F}(H \beta)$ & & & & $-1:$ & & & & & & 13 & 3.3 \\
\hline
\end{tabular}


Table A3 Ionic and total abundances of Hen 2-141 and NGC 5307.

\begin{tabular}{|c|c|c|c|c|c|}
\hline & Hen 2-141 & & NGC & 5307 & \\
\hline & Entire PN & Entire PN & $\mathrm{K}_{\mathrm{NW}}$ & $\mathrm{K}_{\mathrm{SW}}$ & $\mathrm{K}_{\mathrm{SE}}$ \\
\hline $\mathrm{He}^{+} / \mathrm{H}$ & $5.35 \mathrm{E}-02(2.6 \mathrm{E}-3)(-2.6 \mathrm{E}-3)$ & $8.04 \mathrm{E}-02(7.2 \mathrm{E}-3)(-6.6 \mathrm{E}-3)$ & $1.09 \mathrm{E}-01(6.0 \mathrm{E}-3)(-5.5 \mathrm{E}-3)$ & $1.17 \mathrm{E}-01(4.3 \mathrm{E}-3)(-4.3 \mathrm{E}-3)$ & $9.28 \mathrm{E}-02(5.0 \mathrm{E}-3)(-4.8 \mathrm{E}-3)$ \\
\hline $\mathrm{He}^{2+} / \mathrm{H}$ & $5.86 \mathrm{E}-02(5.8 \mathrm{E}-4)(-5.8 \mathrm{E}-4)$ & $2.06 \mathrm{E}-02(3.0 \mathrm{E}-4)(-3.0 \mathrm{E}-4)$ & $1.26 \mathrm{E}-03(9.1 \mathrm{E}-5)(-9.1 \mathrm{E}-5)$ & $6.70 \mathrm{E}-03(1.7 \mathrm{E}-4)(-1.7 \mathrm{E}-4)$ & $1.42 \mathrm{E}-03(8.6 \mathrm{E}-5)(-8.1 \mathrm{E}-5)$ \\
\hline $\mathrm{He} / \mathrm{H}$ & $1.12 \mathrm{E}-01(2.5 \mathrm{E}-3)(-2.5 \mathrm{E}-3)$ & $1.01 \mathrm{E}-01(7.0 \mathrm{E}-3)(-6.6 \mathrm{E}-3)$ & $1.10 \mathrm{E}-01(6.0 \mathrm{E}-3)(-5.5 \mathrm{E}-3)$ & $1.24 \mathrm{E}-01(4.3 \mathrm{E}-3)(-4.3 \mathrm{E}-3)$ & $9.43 \mathrm{E}-02(5.0 \mathrm{E}-3)(-4.8 \mathrm{E}-3)$ \\
\hline $\mathrm{C}^{2+} / \mathrm{H}$ & $6.52 \mathrm{E}-04(1.4 \mathrm{E}-4)(-1.4 \mathrm{E}-4)$ & $1.52 \mathrm{E}-04(2.7 \mathrm{E}-5)(-2.6 \mathrm{E}-5)$ & & $2.52 \mathrm{E}-04(5.3 \mathrm{E}-5)(-5.2 \mathrm{E}-5)$ & \\
\hline $\mathrm{C}^{3+} / \mathrm{H}$ & & $1.43 \mathrm{E}-04(1.8 \mathrm{E}-5)(-1.6 \mathrm{E}-5)$ & $1.89 \mathrm{E}-04(3.5 \mathrm{E}-5)(-3.3 \mathrm{E}-5)$ & $1.51 \mathrm{E}-04(2.1 \mathrm{E}-5)(-2.1 \mathrm{E}-5)$ & $6.40 \mathrm{E}-05(1.7 \mathrm{E}-5)(-1.7 \mathrm{E}-5)$ \\
\hline $\operatorname{icf}(\mathrm{C})$ & $1.03(0.04)(-0.05)$ & $1.20(0.01)(-0.01)$ & $1.00(0.00)(0.00)$ & $1.00(0.00)(0.00)$ & $1.00(0.00)(0.00)$ \\
\hline $\mathrm{C} / \mathrm{H}$ & $6.69 \mathrm{E}-04(2.7 \mathrm{E}-4)(-2.7 \mathrm{E}-4)$ & $3.53 \mathrm{E}-04(3.8 \mathrm{E}-5)(-3.8 \mathrm{E}-5)$ & $1.89 \mathrm{E}-04(3.5 \mathrm{E}-5)(-3.3 \mathrm{E}-5)$ & $4.03 \mathrm{E}-04(5.6 \mathrm{E}-5)(-5.7 \mathrm{E}-5)$ & $6.40 \mathrm{E}-05(1.7 \mathrm{E}-5)(-1.7 \mathrm{E}-5)$ \\
\hline $\begin{array}{l}\mathrm{N}^{+} / \mathrm{H} \\
\mathrm{jcf}(\mathrm{N})\end{array}$ & $1.49 \mathrm{E}-05(3.2 \mathrm{E}-6)(-2.6 \mathrm{E}-6)$ & $9.12 \mathrm{E}-07(2.7 \mathrm{E}-7)(-2.1 \mathrm{E}-7)$ & $\begin{array}{l}8.89 \mathrm{E}-06(1.8 \mathrm{E}-6)(-1.5 \mathrm{E}-6) \\
10.06(2.03)(-1.70)\end{array}$ & $\begin{array}{r}2.04 \mathrm{E}-06(2.4 \mathrm{E}-7)(-2.2 \mathrm{E}-7) \\
48.73(11.41)(-9.25)\end{array}$ & $\begin{array}{c}4.03 \mathrm{E}-06(7.8 \mathrm{E}-7)(-5.9 \mathrm{E}-7) \\
21.83(6.88)(-6.70)\end{array}$ \\
\hline $\mathrm{N} / \mathrm{H}$ & $1.55 \mathrm{E}-04(2.3 \mathrm{E}-5)(-2.1 \mathrm{E}-5)$ & $5.24 \mathrm{E}-05(1.5 \mathrm{E}-5)(-1.2 \mathrm{E}-5)$ & $8.96 \mathrm{E}-05(1.2 \mathrm{E}-5)(-1.2 \mathrm{E}-5)$ & $9.93 \mathrm{E}-05(2.1 \mathrm{E}-5)(-1.8 \mathrm{E}-5)$ & $8.64 \mathrm{E}-05(2.1 \mathrm{E}-5)(-2.0 \mathrm{E}-5)$ \\
\hline $\mathrm{O}^{+} / \mathrm{H}$ & $4.01 \mathrm{E}-05(1.4 \mathrm{E}-5)$ & $6(1.6 \mathrm{H}$ & 6) & 5.76 & $1.41 \mathrm{E}-05(6.2 \mathrm{~F}$ \\
\hline $\mathrm{O}^{2+} / \mathrm{H}$ & $2.76 \mathrm{E}-04(8.5 \mathrm{E}-6)(-8.1 \mathrm{E}-6)$ & $2.38 \mathrm{E}-04(4.3 \mathrm{E}-5)(-3.6 \mathrm{E}-5)$ & $2.12 \mathrm{E}-04(1.6 \mathrm{E}-5)(-1.5 \mathrm{E}-5)$ & $2.42 \mathrm{E}-04(4.2 \mathrm{E}-5)(-3.6 \mathrm{E}-5)$ & $2.17 \mathrm{E}-04(2.3 \mathrm{E}-5)(-2.1 \mathrm{E}-5)$ \\
\hline $\operatorname{icf}(\mathrm{O})$ & $1.65(0.04)(-0.03)$ & $1.14(0.01)(-0.01)$ & $1.01(0.00)(0.00)$ & $1.03(0.00)(0.00)$ & $1.01(0.00)(0.00)$ \\
\hline $\mathrm{O} / \mathrm{H}$ & $5.24 \mathrm{E}-04(2.8 \mathrm{E}-5)(-2.3 \mathrm{E}-5)$ & $2.77 \mathrm{E}-04(4.8 \mathrm{E}-5)(-4.1 \mathrm{E}-5)$ & $2.47 \mathrm{E}-04(1.7 \mathrm{E}-5)(-1.6 \mathrm{E}-5)$ & $2.55 \mathrm{E}-04(4.3 \mathrm{E}-5)(-3.7 \mathrm{E}-5)$ & $2.35 \mathrm{E}-04(2.7 \mathrm{E}-5)(-2.2 \mathrm{E}-5)$ \\
\hline $\mathrm{Ne}^{2+} / \mathrm{H}$ & $6.29 \mathrm{E}-05(3.9 \mathrm{E}-6)(-3.7 \mathrm{E}-6)$ & $5.72 \mathrm{E}-05(5.9 \mathrm{E}-6)(-5.4 \mathrm{E}-6)$ & $7.88 \mathrm{E}-05(4.0 \mathrm{E}-6)(-3.8 \mathrm{E}-6)$ & $6.45 \mathrm{E}-05(6.9 \mathrm{E}-6)(-6.2 \mathrm{E}-6)$ & $5.82 \mathrm{E}-05(7.3 \mathrm{E}-6)$ \\
\hline $\operatorname{icf}(\mathrm{Ne})$ & $1.68(0.05)$ & $1.14(0.01)(-0.01)$ & $1.27(0$. & $1.03(0.0$ & \\
\hline $\mathrm{Ne} / \mathrm{H}$ & $1.06 \mathrm{E}-04(7.4 \mathrm{E}-6)(-6.9 \mathrm{E}-6)$ & $6.54 \mathrm{E}-05(6.7 \mathrm{E}-6)(-6.1 \mathrm{E}-6)$ & $9.99 \mathrm{E}-05(5.0 \mathrm{E}-6)(-4.7 \mathrm{E}-6)$ & $6.67 \mathrm{E}-05(7.1 \mathrm{E}-6)(-6.4 \mathrm{E}-6)$ & $6.78 \mathrm{E}-05(9.3 \mathrm{E}-6)(-7.7 \mathrm{E}-6)$ \\
\hline $\mathrm{Ar}^{2+} / \mathrm{H}$ & & $2.63 \mathrm{E}-07(7.8 \mathrm{E}-8)(-6.8$ & & $4.78 \mathrm{E}-07(1.6 \mathrm{E}-7)(-1$. & $8.17 \mathrm{E}-07(2.1 \mathrm{E}-7)(-1.8 \mathrm{E}-7)$ \\
\hline $\mathrm{Ar}^{3+} / \mathrm{H}$ & $1.14 \mathrm{E}-06(3.4 \mathrm{E}-8)(-3.0 \mathrm{E}-8)$ & $7.03 \mathrm{E}-07(6.7 \mathrm{E}-8)(-6.1 \mathrm{E}-8)$ & $3.04 \mathrm{E}-07(1.8 \mathrm{E}-8)(-1.7 \mathrm{E}-8)$ & $5.14 \mathrm{E}-07(4.9 \mathrm{E}-8$ & $4.43 \mathrm{E}-07(4.3 \mathrm{E}-8)$ \\
\hline $\operatorname{icf}(\operatorname{Ar})$ & $1.00(0.00)(0.00$ & $1.00(0.00)(0.00)$ & $1.00(0.00)(0.00)$ & $1.00(0.00)(0.00)$ & $1.00(0.08)(0.00)$ \\
\hline $\mathrm{Ar} / \mathrm{H}$ & $1.14 \mathrm{E}-06(3.4 \mathrm{E}-8)(-3.0 \mathrm{E}-8)$ & $9.65 \mathrm{E}-07(1.3 \mathrm{E}-7)(-1.1 \mathrm{E}-7)$ & $3.04 \mathrm{E}-07(1.8 \mathrm{E}-8)(-1.7 \mathrm{E}-8)$ & $9.88 \mathrm{E}-07(2.0 \mathrm{E}-7)(-1.7 \mathrm{E}-7)$ & $1.30 \mathrm{E}-06(2.9 \mathrm{E}-7)(-2.4 \mathrm{E}-7)$ \\
\hline $\mathrm{S}^{+} / \mathrm{H}$ & $3.60 \mathrm{E}-07(7.8 \mathrm{E}-8)(-5.5 \mathrm{E}$ & $9.06 \mathrm{E}-08(2.5 \mathrm{E}-8)(-1.8 \mathrm{E}$ & $7.17 \mathrm{E}-07(1.3 \mathrm{E}-7)(-1.0 \mathrm{E}-7)$ & $1.50 \mathrm{E}-07(2.2 \mathrm{E}-8)(-1.9$ & $3.12 \mathrm{E}-07(1.1 \mathrm{E}-7)(-5.1 \mathrm{E}-8)$ \\
\hline $\mathrm{S}^{2+} / \mathrm{H}$ & $2.61 \mathrm{E}-06(1.7 \mathrm{E}-7)(-1.6 \mathrm{E}-7)$ & $1.22 \mathrm{E}-06(2.2 \mathrm{E}-7)(-1.8 \mathrm{E}-7)$ & $4.29 \mathrm{E}-06(4.7 \mathrm{E}-7)(-4.2 \mathrm{E}-7)$ & $1.85 \mathrm{E}-06(2.6 \mathrm{E}-7)(-2.3 \mathrm{E}-7)$ & $2.06 \mathrm{E}-06(2.7 \mathrm{E}-7)(-2.4 \mathrm{E}-7)$ \\
\hline $\operatorname{icf}(\mathrm{S})$ & $2.30(0.21)(-0.20)$ & $2.73(0.29)(-0.23)$ & $1.37(0.09)(-0.08)$ & $2.29(0.12)(-0.11)$ & $1.78(0.13)(-0.18)$ \\
\hline $\mathrm{S} / \mathrm{H}$ & $6.86 \mathrm{E}-06(6.6 \mathrm{E}-7)(-5.9 \mathrm{E}-7)$ & $3.59 \mathrm{E}-06(8.2 \mathrm{E}-7)(-6.7 \mathrm{E}-7)$ & $6.87 \mathrm{E}-06(8.5 \mathrm{E}-7)(-7.5 \mathrm{E}-7)$ & 4.59E-06 ( 7.9E-7 ) (-6.7E-7 ) & $4.23 \mathrm{E}-06(6.8 \mathrm{E}-7)(-5.6 \mathrm{E}-7)$ \\
\hline $\mathrm{Cl}^{2+} / \mathrm{H}$ & $6.81 \mathrm{E}-08(1.0 \mathrm{E}-8)(-9.7 \mathrm{E}-9)$ & $4.94 \mathrm{E}-08(5.8 \mathrm{E}-9)(-5.2 \mathrm{E}-9)$ & $8.64 \mathrm{E}-08(2.2 \mathrm{E}-8)(-2.2 \mathrm{E}-8)$ & $3.44 \mathrm{E}-08(4.3 \mathrm{E}-9)(-3.8 \mathrm{E}-9)$ & $5.10 \mathrm{E}-08(9.6 \mathrm{E}-9)(-9.6 \mathrm{E}-$ \\
\hline $\operatorname{icf}(\mathrm{C}$ & $2.61(0.15)(-0.14)$ & $1.00(0.00)(0.00)$ & $1.56(0.06)(-0.05)$ & $1.00(0.00)(0.00)$ & $1.78(0.11)(-0.78)$ \\
\hline $\mathrm{Cl} / \mathrm{H}$ & $1.78 \mathrm{E}-07(2.8 \mathrm{E}-8)(-2.7 \mathrm{E}-8)$ & $4.95 \mathrm{E}-08(5.9 \mathrm{E}-9)(-5.2 \mathrm{E}-9)$ & $1.35 \mathrm{E}-07(3.6 \mathrm{E}-8)(-3.5 \mathrm{E}-8)$ & $3.44 \mathrm{E}-08(4.3 \mathrm{E}-9)(-3.9 \mathrm{E}-9)$ & $7.84 \mathrm{E}-08(2.9 \mathrm{E}-8)(-2.1 \mathrm{E}-8)$ \\
\hline
\end{tabular}

Table A4 Ionic and total abundances of IC 2553 and PB6.

\begin{tabular}{|c|c|c|c|c|c|}
\hline & & IC 2553 & & $\overline{\mathrm{P} 1}$ & \\
\hline & Entire PN & $\mathrm{K}_{\mathrm{NE}}$ & $\mathrm{K}_{\mathrm{SW}}$ & Entire PN & $\mathrm{K}_{\mathrm{SE}} \mathrm{Knot}$ \\
\hline $\mathrm{He}^{+} / \mathrm{H}$ & $8.80 \mathrm{E}-02(6.0 \mathrm{E}-3)(-5.6 \mathrm{E}-3)$ & $7.02 \mathrm{E}-02(4.3 \mathrm{E}-3)(-4.3 \mathrm{E}-3)$ & $1.01 \mathrm{E}-01(6.5 \mathrm{E}-3)(-6.1 \mathrm{E}-3)$ & $4.67 \mathrm{E}-02(3.2 \mathrm{E}-3)(-3.2 \mathrm{E}-3)$ & $6.64 \mathrm{E}-02(5.4 \mathrm{E}-3)(-5.0 \mathrm{E}-3)$ \\
\hline $\mathrm{He}^{2+} / \mathrm{H}$ & $2.09 \mathrm{E}-02(3.5 \mathrm{E}-4)(-3.5 \mathrm{E}-4)$ & $1.25 \mathrm{E}-02(1.1 \mathrm{E}-3)(-1.1 \mathrm{E}-3)$ & $8.09 \mathrm{E}-03(2.4 \mathrm{E}-4)(-2.4 \mathrm{E}-4)$ & $1.20 \mathrm{E}-01(1.1 \mathrm{E}-3)(-1.1 \mathrm{E}-3)$ & $8.62 \mathrm{E}-02(2.5 \mathrm{E}-3)(-2.5 \mathrm{E}-3)$ \\
\hline $\mathrm{He} / \mathrm{H}$ & $1.09 \mathrm{E}-01(5.9 \mathrm{E}-3)(-5.6 \mathrm{E}-3)$ & $8.27 \mathrm{E}-02(4.4 \mathrm{E}-3)(-4.4 \mathrm{E}-3)$ & $1.09 \mathrm{E}-01(6.4 \mathrm{E}-3)(-6.1 \mathrm{E}-3)$ & $1.67 \mathrm{E}-01(3.2 \mathrm{E}-3)(-3.2 \mathrm{E}-3)$ & $1.53 \mathrm{E}-01(5.7 \mathrm{E}-3)(-5.5 \mathrm{E}-3)$ \\
\hline $\mathrm{C}^{2+} / \mathrm{H}$ & $6.17 \mathrm{E}-04(1.9 \mathrm{E}-5)(-1.9 \mathrm{E}-5)$ & $6.04 \mathrm{E}-04(1.2 \mathrm{E}-4)(-1.2 \mathrm{E}-4)$ & $5.48 \mathrm{E}-04(1.1 \mathrm{E}-4)(-1.1 \mathrm{E}-4)$ & $4.52 \mathrm{E}-04(9.6 \mathrm{E}-5)(-9.2 \mathrm{E}-5)$ & \\
\hline $\mathrm{C}^{3+} / \mathrm{H}$ & $6.20 \mathrm{E}-05(1.2 \mathrm{E}-5)(-1.3 \mathrm{E}-5)$ & & & $2.74 \mathrm{E}-04(4.4 \mathrm{E}-5)(-4.4 \mathrm{E}-5)$ & \\
\hline $\operatorname{icf}(\mathrm{C})$ & $1.19(0.01)(-0.02)$ & $1.09(0.02)(-0.03)$ & $1.07(0.03)(-0.03)$ & $1.00(0.00)(0.00)$ & \\
\hline $\mathrm{C} / \mathrm{H}$ & $8.06 \mathrm{E}-04(3.0 \mathrm{E}-5)(-3.0 \mathrm{E}-5)$ & $6.59 \mathrm{E}-04(1.6 \mathrm{E}-4)(-1.6 \mathrm{E}-4)$ & $5.88 \mathrm{E}-04(1.4 \mathrm{E}-4)(-1.4 \mathrm{E}-4)$ & $7.26 \mathrm{E}-04(1.1 \mathrm{E}-4)(-1.0 \mathrm{E}-4)$ & \\
\hline $\mathrm{N}^{+} / \mathrm{H}$ & $3.84 \mathrm{E}-06(9.8 \mathrm{E}-7)(-6.3 \mathrm{E}-7)$ & $1.22 \mathrm{E}-05(1.5 \mathrm{E}-6)(-1.4 \mathrm{E}-6)$ & $1.21 \mathrm{E}-05(1.5 \mathrm{E}-6)(-1.3 \mathrm{E}-6)$ & $2.51 \mathrm{E}-05(2.3 \mathrm{E}-6)(-2.1 \mathrm{E}-6)$ & $4.12 \mathrm{E}-05(5.3 \mathrm{E}-6)(-4.7 \mathrm{E}-6)$ \\
\hline $\operatorname{icf}(\mathrm{N})$ & $45.06(14.66)(-14.93)$ & $12.69(2.87)(-2.23)$ & $11.77(2.68)(-2.18)$ & $15.04(1.86)(-1.66)$ & $8.99(1.79)(-1.49)$ \\
\hline $\mathrm{N} / \mathrm{H}$ & $1.70 \mathrm{E}-04(4.6 \mathrm{E}-5)(-5.0 \mathrm{E}-5)$ & $1.55 \mathrm{E}-04(2.9 \mathrm{E}-5)(-2.4 \mathrm{E}-5)$ & $1.42 \mathrm{E}-04(2.6 \mathrm{E}-5)(-2.2 \mathrm{E}-5)$ & $3.77 \mathrm{E}-04(2.9 \mathrm{E}-5)(-2.7 \mathrm{E}-5)$ & $3.70 \mathrm{E}-04(5.3 \mathrm{E}-5)(-4.6 \mathrm{E}-5)$ \\
\hline $\mathrm{O}^{+} / \mathrm{H}$ & $7.70 \mathrm{E}-06(3.7 \mathrm{E}-6)(-1.8 \mathrm{E}-6)$ & $2.76 \mathrm{E}-05(5.4 \mathrm{E}-6)(-4.7 \mathrm{E}-6)$ & $3.07 \mathrm{E}-05(6.3 \mathrm{E}-6)(-5.1 \mathrm{E}-6)$ & $1.74 \mathrm{E}-05(2.4 \mathrm{E}-6)(-2.1 \mathrm{E}-6)$ & $2.82 \mathrm{E}-05(6.0 \mathrm{E}-6)(-4.9 \mathrm{E}-6)$ \\
\hline $\mathrm{O}^{2+} / \mathrm{H}$ & $3.33 \mathrm{E}-04(2.4 \mathrm{E}-5)(-2.2 \mathrm{E}-5)$ & $3.11 \mathrm{E}-04(3.8 \mathrm{E}-5)(-3.4 \mathrm{E}-5)$ & $3.01 \mathrm{E}-04(3.5 \mathrm{E}-5)(-3.1 \mathrm{E}-5)$ & $1.14 \mathrm{E}-04(3.3 \mathrm{E}-6)(-3.2 \mathrm{E}-6)$ & $1.50 \mathrm{E}-04(1.8 \mathrm{E}-5)(-1.6 \mathrm{E}-5)$ \\
\hline $\operatorname{icf}(\mathrm{O})$ & $1.13(0.01)(-0.01)$ & $1.10(0.01)(-0.01)$ & $1.04(0.00)(0.00)$ & $2.62(0.12)(-0.12)$ & $1.78(0.07)(-0.07)$ \\
\hline $\mathrm{O} / \mathrm{H}$ & $3.91 \mathrm{E}-04(3.1 \mathrm{E}-5)(-2.8 \mathrm{E}-5)$ & $3.73 \mathrm{E}-04(4.3 \mathrm{E}-5)(-3.9 \mathrm{E}-5)$ & $3.47 \mathrm{E}-04(3.7 \mathrm{E}-5)(-3.3 \mathrm{E}-5)$ & $3.44 \mathrm{E}-04(2.0 \mathrm{E}-5)(-1.7 \mathrm{E}-5)$ & $3.19 \mathrm{E}-04(3.3 \mathrm{E}-5)(-3.0 \mathrm{E}-5)$ \\
\hline $\mathrm{Ne}^{2+} / \mathrm{H}$ & $9.08 \mathrm{E}-05(4.4 \mathrm{E}-6)(-4.4 \mathrm{E}-6)$ & $1.17 \mathrm{E}-04(1.9 \mathrm{E}-5)(-1.7 \mathrm{E}-5)$ & $1.19 \mathrm{E}-04(1.6 \mathrm{E}-5)(-1.4 \mathrm{E}-5)$ & $3.21 \mathrm{E}-05(9.4 \mathrm{E}-7)(-9.2 \mathrm{E}-7)$ & $4.64 \mathrm{E}-05(6.5 \mathrm{E}-6)(-5.7 \mathrm{E}-6)$ \\
\hline $\operatorname{icf}(\mathrm{Ne})$ & $1.13(0.01)(-0.01)$ & $1.10(0.01)(-0.01)$ & $1.05(0.00)(0.00)$ & $3.12(0.23)(-0.22)$ & $1.85(0.10)(-0.09)$ \\
\hline $\mathrm{Ne} / \mathrm{H}$ & $1.03 \mathrm{E}-04(5.3 \mathrm{E}-6)(-5.0 \mathrm{E}-6)$ & $1.29 \mathrm{E}-04(2.1 \mathrm{E}-5)(-1.8 \mathrm{E}-5)$ & $1.24 \mathrm{E}-04(1.7 \mathrm{E}-5)(-1.5 \mathrm{E}-5)$ & $9.96 \mathrm{E}-05(8.7 \mathrm{E}-6)(-7.1 \mathrm{E}-6)$ & $8.56 \mathrm{E}-05(1.2 \mathrm{E}-5)(-1.0 \mathrm{E}-5)$ \\
\hline $\mathrm{Ar}^{2+} / \mathrm{H}$ & $1.07 \mathrm{E}-06(9.8 \mathrm{E}-8)(-9.0 \mathrm{E}-8)$ & $1.20 \mathrm{E}-06(2.3 \mathrm{E}-7)(-1.9 \mathrm{E}-7)$ & & & \\
\hline $\mathrm{Ar}^{3+} / \mathrm{H}$ & $1.36 \mathrm{E}-06(6.0 \mathrm{E}-8)(-5.7 \mathrm{E}-8)$ & $6.76 \mathrm{E}-07(8.6 \mathrm{E}-8)(-7.6 \mathrm{E}-8)$ & $5.78 \mathrm{E}-07(7.7 \mathrm{E}-8)(-6.8 \mathrm{E}-8)$ & 8.99E-07 ( $3.2 \mathrm{E}-8)(-2.7 \mathrm{E}-8)$ & $9.06 \mathrm{E}-07(1.2 \mathrm{E}-7)(-1.0 \mathrm{E}-7)$ \\
\hline $\operatorname{icf}(\mathrm{Ar})$ & $1.00(0.00)(0.00)$ & $1.04(0.06)(-0.04)$ & $1.00(0.00)(0.00)$ & $1.00(0.00)(0.00)$ & $1.00(0.00)(0.00)$ \\
\hline $\mathrm{Ar} / \mathrm{H}$ & $2.42 \mathrm{E}-06(1.5 \mathrm{E}-7)(-1.4 \mathrm{E}-7)$ & $1.98 \mathrm{E}-06(3.9 \mathrm{E}-7)(-3.2 \mathrm{E}-7)$ & $5.78 \mathrm{E}-07(7.7 \mathrm{E}-8)(-6.8 \mathrm{E}-8)$ & 8.99E-07 ( $3.2 \mathrm{E}-8)(-2.7 \mathrm{E}-8)$ & $9.06 \mathrm{E}-07(1.2 \mathrm{E}-7)(-1.0 \mathrm{E}-7)$ \\
\hline $\mathrm{S}^{+} / \mathrm{H}$ & $2.61 \mathrm{E}-07(1.1 \mathrm{E}-7)(-3.7 \mathrm{E}-8)$ & $5.54 \mathrm{E}-07(6.7 \mathrm{E}-8)(-5.7 \mathrm{E}-8)$ & $7.26 \mathrm{E}-07(9.6 \mathrm{E}-8)(-8.0 \mathrm{E}-8)$ & $3.61 \mathrm{E}-07(3.4 \mathrm{E}-8)(-3.1 \mathrm{E}-8)$ & $5.23 \mathrm{E}-07(6.1 \mathrm{E}-8)(-5.4 \mathrm{E}-8)$ \\
\hline $\mathrm{S}^{2+} / \mathrm{H}$ & $3.71 \mathrm{E}-06(4.9 \mathrm{E}-7)(-4.3 \mathrm{E}-7)$ & $2.87 \mathrm{E}-06(5.0 \mathrm{E}-7)(-4.2 \mathrm{E}-7)$ & $4.39 \mathrm{E}-06(7.6 \mathrm{E}-7)(-6.5 \mathrm{E}-7)$ & $1.77 \mathrm{E}-06(9.2 \mathrm{E}-8)(-9.2 \mathrm{E}-8)$ & $1.70 \mathrm{E}-06(3.1 \mathrm{E}-7)(-2.6 \mathrm{E}-7)$ \\
\hline $\operatorname{icf}(\mathrm{S})$ & $2.53(0.18)(-0.22)$ & $1.78(0.11)(-0.11)$ & $1.62(0.11)(-0.10)$ & $3.59(0.23)(-0.21)$ & $2.30(0.18)(-0.16)$ \\
\hline $\mathrm{S} / \mathrm{H}$ & $9.96 \mathrm{E}-06(1.7 \mathrm{E}-6)(-1.5 \mathrm{E}-6)$ & $6.10 \mathrm{E}-06(1.1 \mathrm{E}-6)(-9.4 \mathrm{E}-7)$ & $8.30 \mathrm{E}-06(1.6 \mathrm{E}-6)(-1.3 \mathrm{E}-6)$ & $7.64 \mathrm{E}-06(5.5 \mathrm{E}-7)(-5.2 \mathrm{E}-7)$ & $5.13 \mathrm{E}-06(8.8 \mathrm{E}-7)(-7.5 \mathrm{E}-7)$ \\
\hline $\mathrm{Cl}^{2+} / \mathrm{H}$ & 8.33E-08 ( 8.0E-9 ) (-7.3E-9 ) & $7.61 \mathrm{E}-08(9.4 \mathrm{E}-9)(-8.3 \mathrm{E}-9)$ & $7.94 \mathrm{E}-08(9.6 \mathrm{E}-9)(-8.6 \mathrm{E}-9)$ & 4.03E-08 ( 7.9E-9 ) ( -7.4E-9) & 3.47E-08 ( $5.1 \mathrm{E}-9)(-4.4 \mathrm{E}-9)$ \\
\hline $\operatorname{icf}(\mathrm{Cl})$ & $1.00(0.00)(0.00)$ & $1.90(0.09)(-0.08)$ & $1.76(0.08)(-0.07)$ & $4.09(0.21)(-0.20)$ & $2.69(0.14)(-0.13)$ \\
\hline $\mathrm{Cl} / \mathrm{H}$ & $8.46 \mathrm{E}-08(1.2 \mathrm{E}-8)(-8.0 \mathrm{E}-9)$ & $1.45 \mathrm{E}-07(2.1 \mathrm{E}-8)(-2.0 \mathrm{E}-8)$ & $1.40 \mathrm{E}-07(2.1 \mathrm{E}-8)(-1.8 \mathrm{E}-8)$ & $1.65 \mathrm{E}-07(3.6 \mathrm{E}-8)(-3.3 \mathrm{E}-8)$ & $9.35 \mathrm{E}-08(1.6 \mathrm{E}-8)(-1.4 \mathrm{E}-8)$ \\
\hline
\end{tabular}



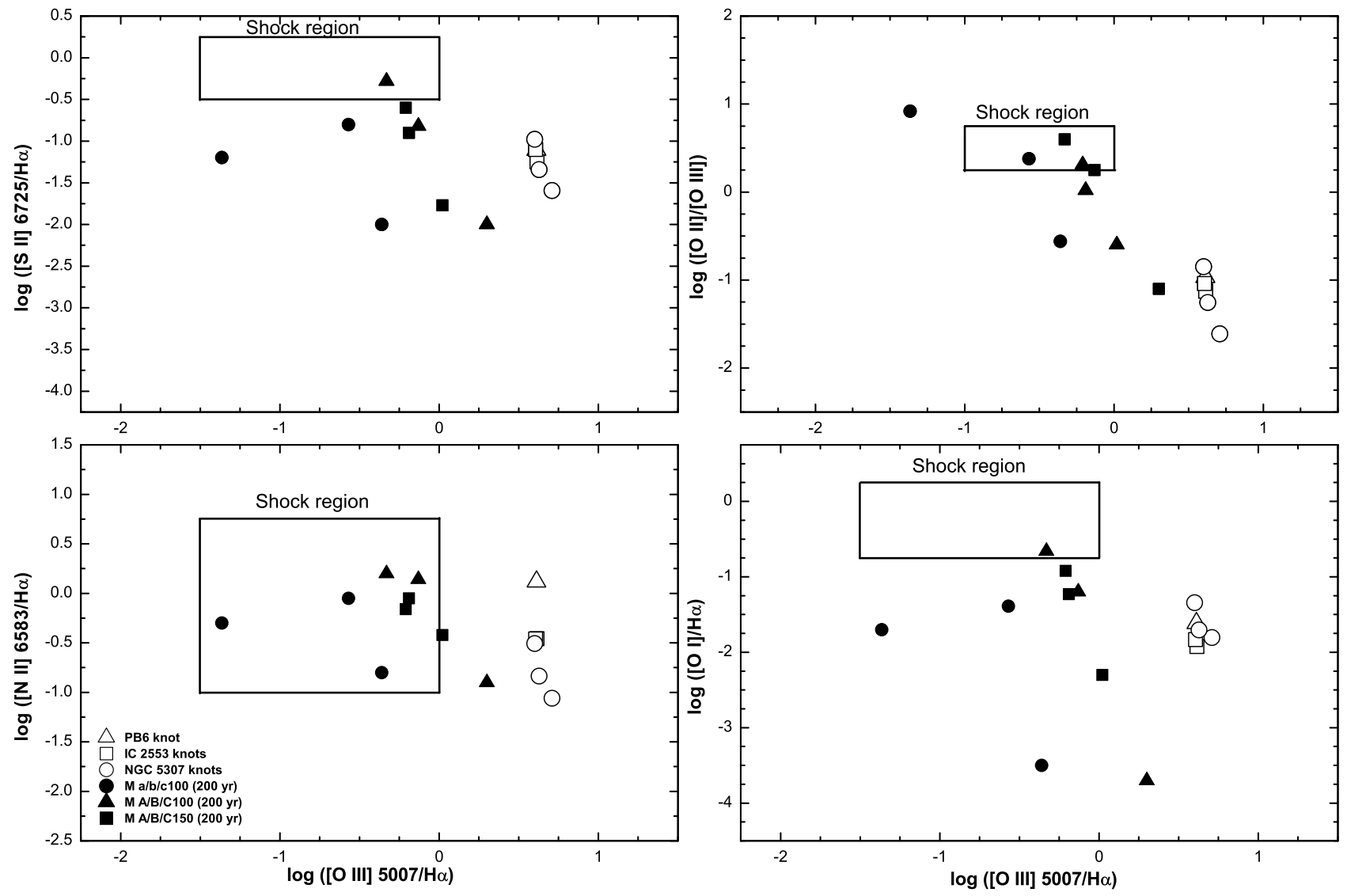

Figure 1. The locations of the knots on the diagnostic diagrams proposed by Raga et al. (2008) designed to distinguish the shockexcited and photoionized regions. The empty symbols refer to the knots associated with PB 6 (triangle), IC 2553 (squares), and NGC 5307 (circles). The rectangle in each diagram refers to the shock region. The filled symbols represent the predicted emission line ratios by Raga et al. (2008); filled circles $=$ models a/b/c/100 ( $\mathrm{t}=200 \mathrm{yr}$ ); filled triangles $=$ models A/B/C/100 ( $\mathrm{t}=200 \mathrm{yr}$ ); filled squares $=$ models $\mathrm{A} / \mathrm{B} / \mathrm{C} / 150(\mathrm{t}=200 \mathrm{yr})$. All knots are far away from the shock-excited regions. This implies that the excitation mechanism responsible for producing these LISs is more likely to be photoionisation from the CSs of their nebulae. 

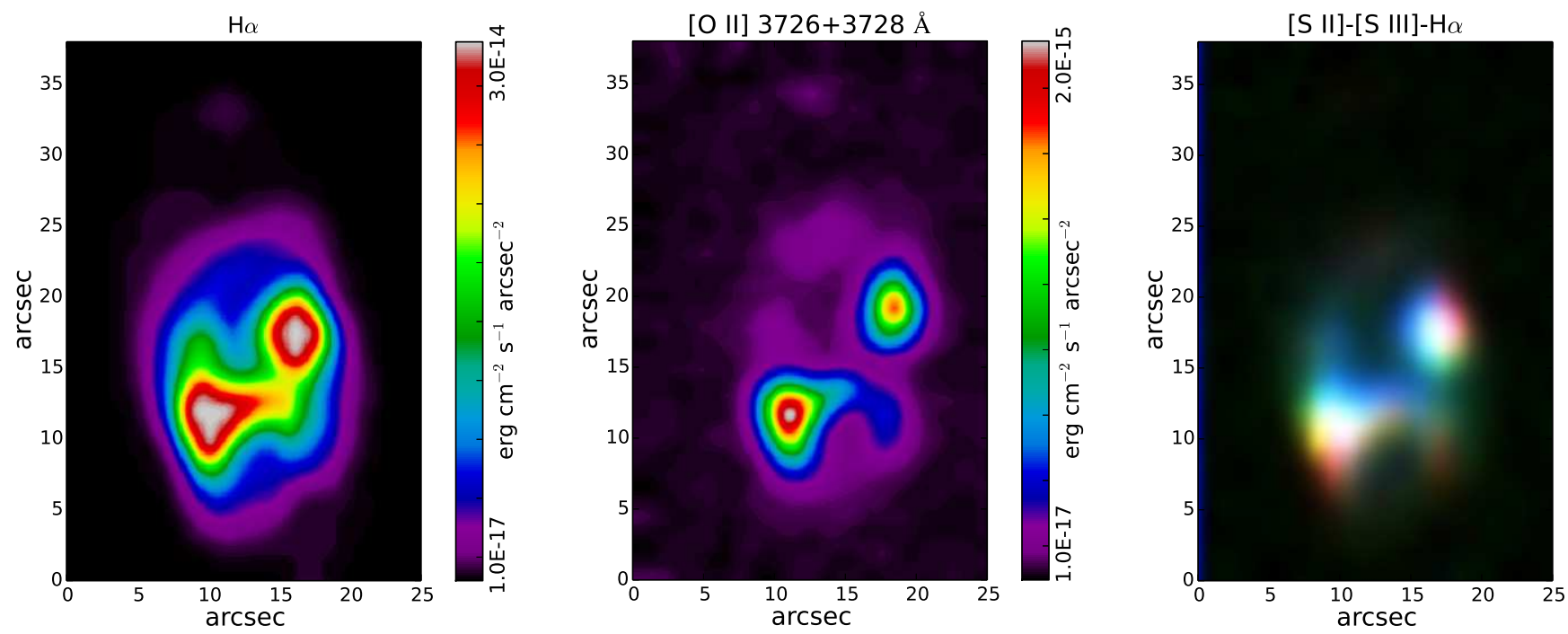

Figure 2. Two emission-line maps in different ionization states and composite color image for Hen 2-141. The left and middle panels show the maps of Hen 2-141 in H $\alpha$ and [O II] 3726+3728 $\AA$ lines, respectively. The color bar in each figure refers to the intensity of emission line through the entire nebula. The top and bottom labels on the color bar represent the maximum and minimum emission values and the scale is linear. The right panel shows a [S II]-[S III]-H $\alpha$ RGB color image of the object. In this and subsequent figures, north is up and east is on the left. This figure show a bipolar morphology for the object with two knots to the north and to the south which barely appear in magenta $(\mathrm{R}+\mathrm{B})$ color (right panel) at the outer limb of the two lobes. The low-ionisation knots are bi-symmetric with respect to the nebular centre.
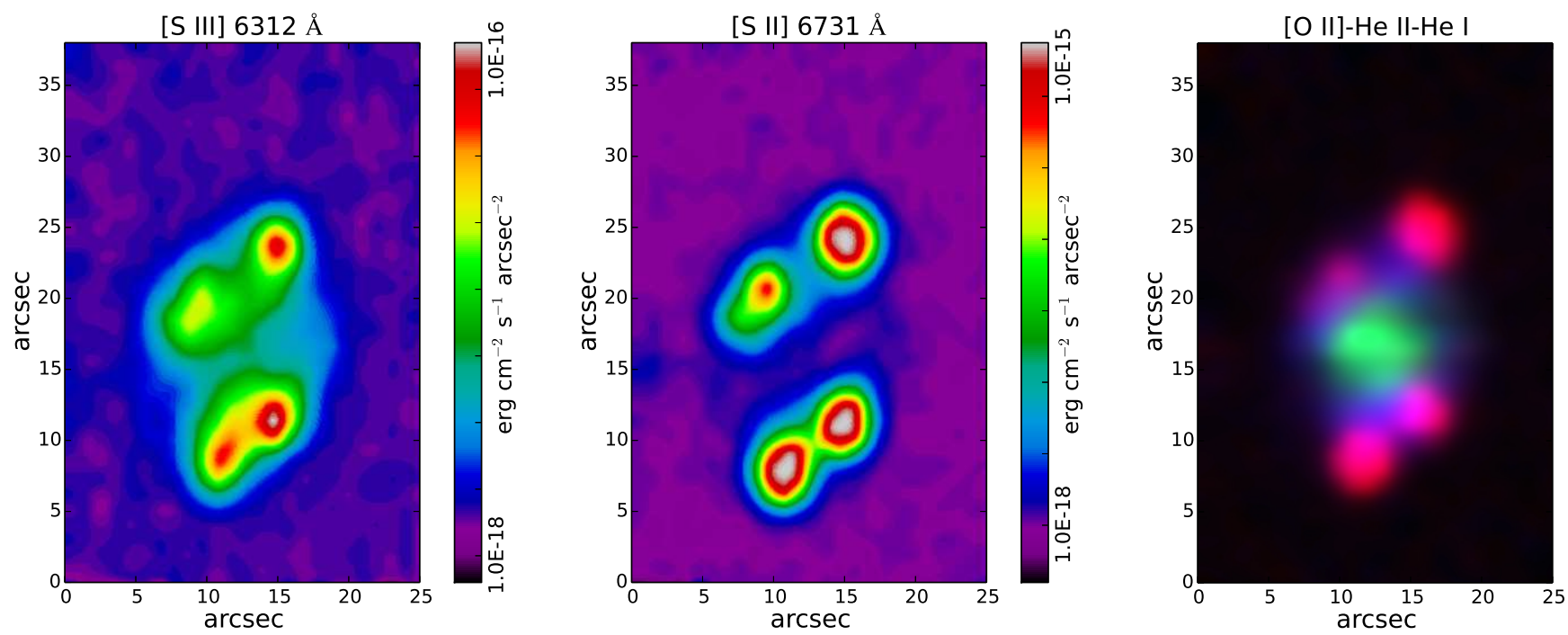

Figure 3. As Fig. 2, but for NGC 5307. The left and middle panels show the maps of NGC 5307 in [S III] and [S II] lines, respectively. The right panel shows an [O II]-He II-He I RGB color image of the object. This object shows a bi-symmetric shape with two pairs of knots on either sides of the nebular center. These four knots are very pronounced in magenta color ([O II] $+\mathrm{He} I)$ in the composite RGB image (right panel). 

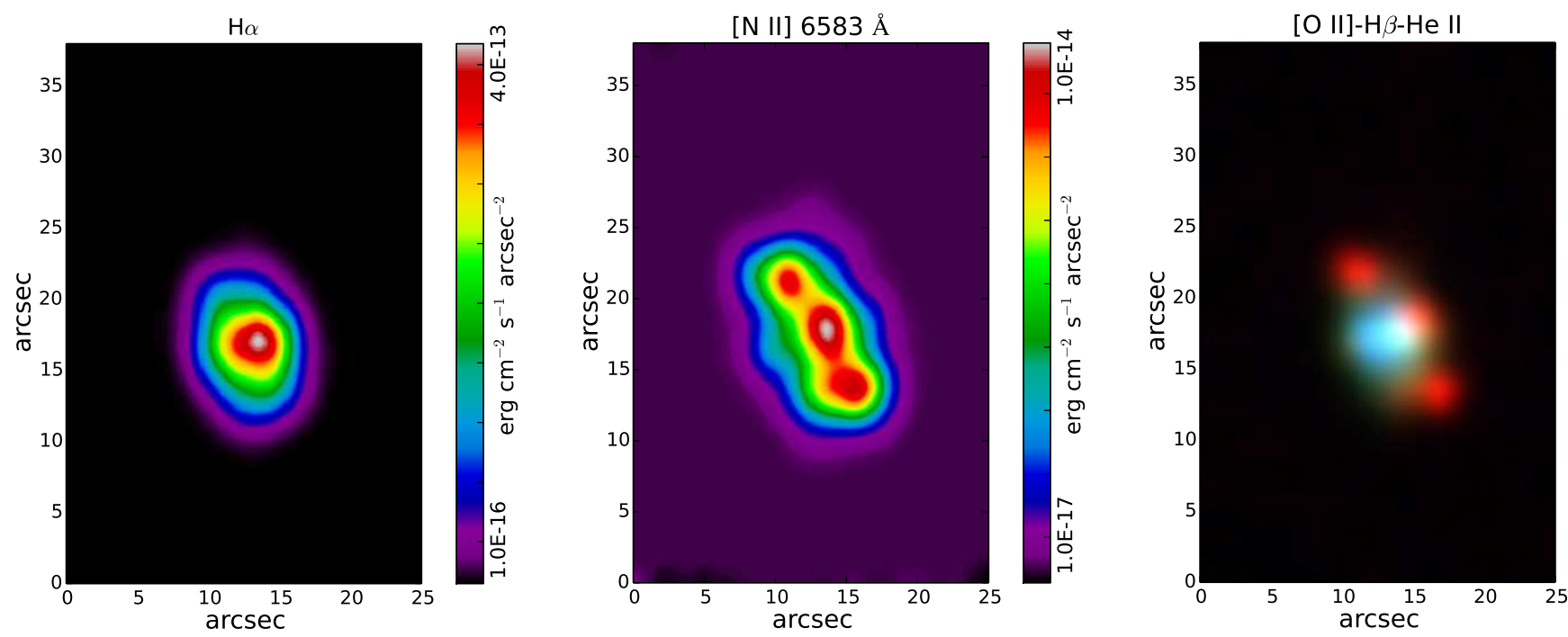

Figure 4. As Fig. 2, but for IC 2553. The left and middle panels show the maps of IC2553 in the H $\alpha$ and [N II] $3726+3728 \AA$ lines, respectively. The right panel shows a [O II]-H $\beta$-He II RGB composite color image of the object. The object show an elliptical morphology with two isolated knots (in red color - right panel) lie symmetrically along the major axis. Further, there is another remarkable low-excitation knot (seen in red) on the outer limb of the central spherical region in the north-west direction.
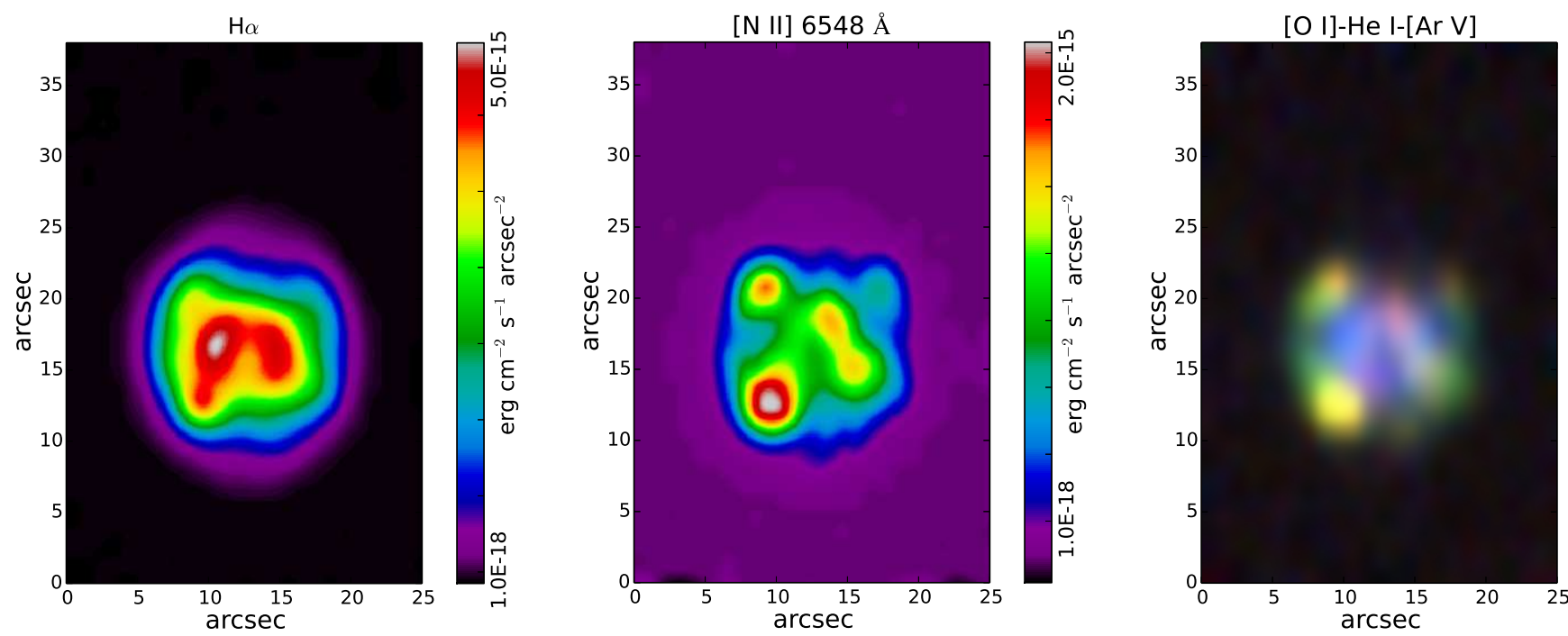

Figure 5. As Fig. 2, but for PB 6. The left and middle panels show the maps of the nebula in H $\alpha$ and [N II] lines, respectively. The right panel shows an [O I] - He I - [Ar V] RGB color image of the object. A roughly circular PN morphology with faint extended halo (appears in [N II] maps) and three non symmetric knots appear inside the nebula. Two bright knots are pronounced in yellow $(\mathrm{R}+\mathrm{G})$ color and the third knot appears in magenta $(\mathrm{R}+\mathrm{B})$ color with a tail of magenta and green colors 